\title{
Postdemokratie, Mitbestimmung und industrielle Bürgerrechte
}

\author{
Ulrich Brinkmann / Oliver Nachtwey
}

\section{Post-Democracy, Co-determination and Industrial Citizenship}

Abstract: The text analyses to what extent the diagnoses of post-democracy (Crouch 2008) can be applied to the field of co-determination in German industrial relations. The main thesis is that co-determination remains stable in formal terms, but is eroding endogenously. Co-determination is organized through democratic procedures; however, a company is not ruled by democracy. For this reason the concept of "industrial citizenship" (T.H. Marshall) is employed to analyze the patterns of democratic participation rights. Using the example of temporary agency work, the changing patterns of industrial citizenship are examined. Afterwards it is shown empirically that the strategic use of temporary agency work and service contracting affects resources and range of co-determination negatively.

Keywords: Co-determination, post-democracy, industrial citizenship, temporary agency work, service contracting

Schlagwörter: Mitbestimmung, Postdemokratie, industrielle Bürgerrechte, Leiharbeit, Werkvertrag

\section{Einleitung}

Colin Crouch geht in seiner Diagnose der „Postdemokratie“ davon aus, dass die Verfahren der Demokratie zwar formal intakt bleiben, aber informell ausgehöhlt werden. Die politischen Entscheidungen werden von der Legitimation der Bürger entkoppelt und stattdessen durch Experten, ökonomische Eliten und ihre Interessen dominiert (Crouch 2008). Allerdings beschränkten die sich auf Crouchs Diagnose folgenden Untersuchungen bisher primär auf das politische Feld. Dieser Beitrag überprüft, ob sich auch in den Industriellen Beziehungen ein Prozess der Postdemokratisierung ausmachen lässt. Postdemokratisierung in den Industriellen Beziehungen heißt nicht, dass die betriebliche Mitbestimmung ihre Funktion als Interessenvertretung der Beschäftigten verliert. Aber sie erodiert endogen - getrieben von der neuen Unternehmenssteuerung im Finanzmarktkapitalismus und darin verschränkten Hybridisierung der industriellen Staatsbürgerrechte.

Zur politischen Ökonomie der westdeutschen Nachkriegsdemokratie gehörten über Jahrzehnte stabile Anerkennungsbeziehungen, institutionalisierte Konfliktregulierungen und verschiedene Varianten tripartistischer Kooperation von Unternehmen, Gewerkschaften und Staat (Streeck u. Hassel 2003). Darüber hinaus gelten die betriebliche Mitbestimmung, Unternehmensmitbestimmung und die Tarifautonomie nicht nur als Element der demokratischen Teilhabe am Wirtschaftsleben, sondern auch als produktiver, stabilisierender und innovativer Faktor der Entwicklung der sozialen Marktwirtschaft (Benthin u. Brinkmann 2008; 
Müller-Jentsch 2011; Streeck u. Kluge 1999). Die Mitbestimmung gehört damit zu den elementaren Bestandteilen der Kompromiss- und Legitimationsproduktion der Sozialordnung in Deutschland (Schroeder u. Weinert 1999).

Hinter der Fassade der Stabilität kriselt das Gesamtsystem der Industriellen Beziehungen, ablesbar etwa an der sinkenden Flächentarifbindung oder an seinem Kernelement der Mitbestimmung. Zurzeit werden noch 44 Prozent der Beschäftigten in den Privatunternehmen West- und 36 Prozent Ostdeutschlands von einem Betriebsrat vertreten (vgl. Abbildung 1):

\section{Abbildung 1: Beschäftigte mit Betriebsrat nach Lage 1993-2011 (in \%)'}

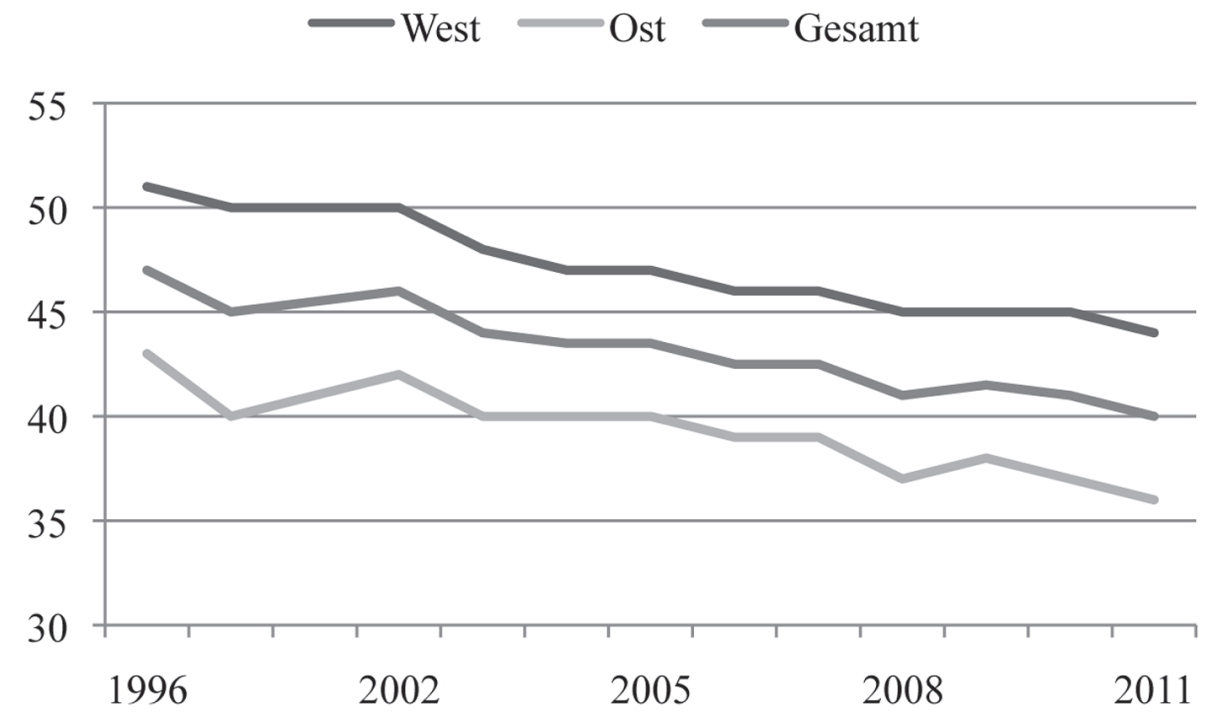

Quelle: IAB-Betriebspanel 2011; eigene Darstellung.

Aber hier ist auf lange Sicht ein abnehmender Deckungsgrad festzustellen. Die Zonen ohne gesetzlich abgesicherte Mitbestimmung wachsen vor allem in den neuen Industrien, aber auch in traditionellen Bereichen und im Dienstleistungssektor kontinuierlich. ${ }^{2}$ Mit anderen Worten: Die Reichweite der institutionalisierten demokratischen Mitbestimmung schwindet.

Der vorliegende Beitrag untersucht, wie sich der demokratische Gehalt der Mitbestimmung in den Zonen der institutionalisierten Mitbestimmung - dort also, wo Betriebsratsgremien vorhanden sind - verändert hat. Dazu wird die These vertreten, dass die Mitbestimmung dem Muster folgt, das W. Streeck (2009a) für die zentralen Institutionen des deutschen Kapitalismus ausgemacht hat: Sie

1 Privatwirtschaftliche Betriebe mit mindestens fünf Beschäftigten ohne Landwirtschaft und Organisationen ohne Erwerbszweck.

$2 \mathrm{Zu}$ den betrieblichen Interessenvertretungen jenseits der institutionalisierten Mitbestimmung vgl. Hauser-Ditz et al. (2008) und Artus (2008). 
erodieren im Verlauf ihrer „normalen“ Entwicklung. In der Mitbestimmung lassen sich trotz formaler Stabilität der Institutionen - und hier besteht die Analogie zum Postdemokratietheorem - eine Erosion sowie eine Fragmentierung der demokratischen Mitbestimmungsstrukturen feststellen.

Crouch analysiert in seinem Essay mehrere Faktoren, die zu einer Postdemokratisierung beitragen: Zentral für die in diesem Aufsatz verfolgte Fragestellung sind (1) der Macht- und Einflussgewinn von Unternehmen (2008, S. 45 ff.). (2) Es sinkt der Einfluss der (organisierten) Arbeiterschaft (2008, S. 71 ff.). (3) Zudem gibt es neue soziale Ungleichheiten, die mit einem Einflusszugewinn für die Eliten und Privilegierten und einem Einflussverlust für die Nicht-Eliten verbunden sind (2008, S. 71 ff.). (4) Negative Freiheitsrechte (Eigentumsrechte etc.) werden gestärkt, während positive Freiheitsrechte - sich zu organisieren und Einfluss auszuüben - an Einfluss verlieren (2008, S. 22).

Die Prozesse in den Industriellen Beziehungen können nicht vollständig analog zu den Faktoren von Crouch analysiert werden. Gleichwohl resultiert der Machtzugewinn der Unternehmen (1) vor allem aus den neuen Unternehmensteuerungsformen, die häufig einen Einflussverlust der Beschäftigten (2) durch die Prekarisierung der Arbeit bedeuten (3). Diese Prozesse werden strukturiert über den Wandel der industriellen Staatsbürgerrechte, der auch die politischen Teilhaberechte und -möglichkeiten vor allem von prekär Beschäftigten beschneidet (4).

Im zweiten Teil des Aufsatzes wird in Anlehnung an den Ansatz von T. H. Marshall rekonstruiert, wie industrielle Staatsbürgerrechte die demokratischen Mitbestimmungsmöglichkeiten in der Betriebsverfassung konstituieren. Der aktuelle Wandel der industriellen Staatsbürgerrechte wurde begleitet durch die Liberalisierungspolitik des Arbeitsmarktes im Zuge der Hartz-Reformen und, so argumentieren wir anschließend, durch neue Formen der Unternehmenssteuerung in den letzten Jahrzehnten vorangetrieben. Dieser Wandel der industriellen Staatsbürgerrechte wird anhand der Sozialfigur des Leiharbeiters erläutert. Die Frage, ob und wie der gestiegene betriebliche Einsatz atypischer Beschäftigung (Leiharbeit und auch Werkverträge) die Ressourcen der Mitbestimmungsakteure neuerdings immer öfter erodieren lässt - während gleichzeitig die Aufgaben der Mitbestimmungsakteure zunehmen -, wird im vierten Abschnitt auf der Basis quantitativen (WSI-Betriebsrätebefragung) und qualitativen Datenmaterials (Betriebsfallstudie) beantwortet. Das Fazit diskutiert die Ergebnisse - auch vor dem Hintergrund des jüngsten Urteils des Bundesarbeitsgerichts zur Leiharbeit, das auf die von uns analysierten Prozesse bereits zum Teil reagiert.

\section{Industrielle Bürgerrechte und Mitbestimmung}

\subsection{Staatsbürgerrechte bei T. H. Marshall}

Staatsbürgerrechte sind ein Produkt der Moderne und des modernen Nationalstaats. Der englische Soziologe T. H. Marshall diagnostizierte in seinem klassischen Beitrag „Staatsbürgerrechte und soziale Klassen“ ([1950] 1992) die Herausbildung von sogenannten sozialen Staatsbürgerrechten. Durch die Entwicklung des Wohlfahrtsstaates der Nachkriegszeit, in Deutschland wie in vielen Ländern 
Europas, transformierte sich auch die Bedeutung der Bürgerrechte. Soziale Bürgerrechte stellte Marshall in eine Linie von (damals) 250 Jahren „kontinuierlichen Fortschritts" der Verallgemeinerung von Staatsbürgerrechten (Marshall 1992, S. 73). In seiner Darstellung entwickeln sich die Bürgerrechte evolutionär in einer aufeinander aufbauenden Stufenleiter; ein einmal erlangtes Bürgerrecht stellte die Grundlage für die Entfaltung des nächsten dar.

Die erste Gruppe der Staatsbürgerrechte umfasste für Marshall die bürgerlichen bzw. zivilen Rechte im Gefolge der bürgerlichen Revolutionen: Rede-, Gedankenund Glaubensfreiheit, die freie Berufswahl, die Vertragsfreiheit, das Recht auf Eigentum und die Einführung einer Gesetzlichkeit. Die zweite, zumeist später verallgemeinerte Gruppe von Bürgerrechten waren die politischen Rechte. Sie umfassten die Rechte auf Teilhabe und Einflussnahme an der politischen Macht. Insbesondere die Entwicklung freier und geheimer Wahlen sowie das allgemeine Wahlrecht bildeten den Kern der politischen Rechte. Die dritte Gruppe, die sozialen Bürgerrechte, garantierten jedem Bürger, jedem Mitglied der Gesellschaft „ein Mindestmaß an wirtschaftlicher Wohlfahrt und Sicherheit" (Marshall 1992, S. 74). Soziale Bürgerechte im Wohlfahrtsstaat waren für Marshall - und die darauf folgenden Diskussionen - jenes gesellschaftliche Element, welches das Spannungsverhältnis zwischen der politischen Gleichheit der Bürger im demokratischen Staat und der sozialen Ungleichheit der Marktvergesellschaftung integrativ zusammenführen konnte (Turner 1986; Lockwood 1996; Mackert 2006).

Marshalls Ansatz wurde vielfach rezipiert und weitergeführt. Er fand Einzug sowohl in die konfliktsoziologischen Arbeiten von Dahrendorf $(1957,1992)$ bis hin zum Strukturfunktionalismus von Parsons ([1977] 2000, 1985), der ebenfalls den evolutionistischen Optimismus Marshalls teilte. Die Kritik, die sich an seinem Ansatz entzündete, bezog sich vor allem auf den Charakter und die Entstehungsbedingungen der (sozialen) Staatsbürgerrechte. Während etwa M. Mann ([1987] 2000) die Gewährung von Staatsbürgerrechten als Legitimations- und Integrationsstrategien der „herrschenden Klassen “ in verschieden Staatsformen betrachtete, betonte Giddens ([1983] 2000; Turner 1990), dass soziale Bürgerrechte vor allem aus gesellschaftlichen Konflikten entstanden waren. In der Konsequenz bedeutet dieses Verständnis, dass sie im Unterschied zu den Menschenrechten nicht per se universalisierbar, sondern in die Form eines (Gesellschafts-) Vertrags gefasst sind (Ganßmann 1993; Tilly 1997).

Bereits gegen Ende des 20. Jahrhunderts haben Reformen eingesetzt, die in den entwickelten Wohlfahrtsstaaten die Marktprinzipien gegenüber den Bürgerrechten wieder aufwerteten (Crouch 1998b.). ${ }^{3}$ Die Ursachen sind dabei vielfältig: die Globalisierung und ihre Folgen für die Nationalstaaten, technologischer und ökonomischer Wandel. Mit dem Jahrtausendwechsel dominieren mittlerweile Diagnosen von einer allgemeinen Erosion der (sozialen) Staatsbürgerschaft (Joppke 2007, 2010; Streeck 2009b; Turner 2001).

3 Mehr implizit als explizit ist dies auch in den Diskussionen in den Analysen zu den Reformen des Wohlfahrtstaates und zu prekärer Beschäftigung zu finden (Lessenich 2008; Castel 2011; Castel u. Dörre 2009). 


\subsection{Industrielle Staatsbürgerrechte}

„Unter der Hand“ (Müller-Jentsch 2008, S. 18) führte Marshall noch den Begriff der industriellen/wirtschaftlichen Bürgerrechte (industrial citizenship) ein. Diese greifen am gleichen Punkt wie die sozialen Bürgerrechte an: dem Spannungsverhältnis zwischen der demokratischen Gleichheit der Bürger und ihrer ökonomischen Ungleichheit. Aber ihren Ausgangspunkt haben sie für Marshall bei den zivilen Bürgerrechten.

Während die zivilen Bürgerrechte dem Individuum Freiheitsrechte gegenüber dem Staat einräumen, der politischen Einflussnahme auf den Staat erlauben, begründen die sozialen Bürgerrechte Ansprüche an den Staat. Im Unterschied hierzu können Gewerkschaften die industriellen Bürgerrechte der Beschäftigten - ihre zivilen, politischen und sozialen Anrechte - in relativer Unabhängigkeit vom Staat in der Tarifarena geltend machen (Barbalet 1998, S. 22-27; Roche 1987, S. 388). Obwohl Marshall sie analog zu den zivilen Bürgerechten, „parallel und ergänzend zum System politischer Staatsbürgerrechte“ ansiedelt (Marshall, S. 82), kombinieren sie in einem komplexen Prozess Elemente der zivilen, politischen und sozialen Bürgerrechte.

Auch wenn industrielle Bürgerrechte die Tarifautonomie (bzw. im angelsächsischen Fall: collective bargaining) und damit auch eine gewisse Akzeptanz des Marktes voraussetzen, sind sie - wie die anderen Bürgerrechte auch - nicht zur Verbesserung ökonomischer Effizienz geschaffen worden (Streeck 2009b). Sie bilden Rechte gegenüber dem Markt, stellen Schutz vor illegitimen hierarchischen und politischen Unterordnungen dar. Unter industriellen Bürgerrechten werden die individuellen, aber vor allem die Kollektivrechte von Arbeitnehmern in der ökonomischen Sphäre zusammengefasst. Wie die anderen Bürgerrechte können sie sowohl einen legalen Status, gesetzlichen Regelungen aber auch ein Set von Konventionen und Praktiken sein, die selbst normativ geteilte Inhalte haben (Fudge 2005, S. 633-634). Sie reichen von den klassischen Arbeitnehmerrechten wie Koalitionsfreiheit über die Möglichkeit zur Partizipation bis hin zum Grad der Dekommodifizierung der Ware Arbeitskraft, z. B. durch die gesetzliche Allgemeinverbindlichkeitserklärung von Tarifnormen (Müller-Jentsch 2007; Streeck 1997; Crouch 1998a; Barbalet 1988; Gersuny 1994; Rees 1996). Auch das über den Arbeitsvertrag geregelte Beschäftigungsverhältnis unterliegt industriellen Bürgerrechten: Das Recht auf Sicherheit am Arbeitsplatz und Gesundheitsschutz, Kündigungsschutz, das Recht auf Urlaub und Erholung, ein soziales Minimum im Krankheitsfall und natürlich das Recht auf eine autonome Repräsentation der Arbeitnehmerinteressen (vgl. Crouch 1998a: S. 152).

Aber industrielle Bürgerrechte haben einen potenziellen Doppelcharakter und können sowohl Element der Integration als auch Grundlage neuer Konflikte sein. Sie können mit anderen Bürgerrechten konfligieren und deshalb nicht einfach als Unterfall der zivilen Bürgerrechte behandelt werden: Der Arbeitsvertrag etwa ist durch die zivilen Bürgerrechte garantiert, er erlaubt dem Arbeitgeber in der mikropolitischen Sphäre der Ökonomie sein Machtdifferential durch Managementkontrolle auszuüben, während auf der anderen Seite die Ausübung von industriellen Bürgerrechten durch die Gewerkschaften - etwa Streiks - sowohl die 
Vertragsfreiheit als auch die managerialen Prärogative des Unternehmers einschränken können (Giddens [1983] 2000, S. 189 ff.).

Industrielle Bürgerrechte sind folglich von ihrer Natur aus „hybrider“ als die anderen Formen der Staatsbürgerrechte (Coutu 2004, S. 75). Sie gründen sich weniger auf einen Bürgerstatus des Individuums, sondern sind Kollektivrechte der Arbeiterschaft, die aus dem Konflikt der Gewerkschaften mit Arbeitgebern, ihren Verbänden und Staat erlangt wurden. Von daher sind sie auch weniger institutionalisiert als die anderen Kategorien der Bürgerrechte; Marshall analysierte sie deshalb als ein durch die Gewerkschaften geschaffenes System „sekundärer, wirtschaftlicher Staatsbürgerschaft" (Marshall 1992, S. 64). Anders als in den voluntaristischen Systemen der britischen Arbeitsbeziehungen, das Marshall vor Augen hatte, sind die industrielle Bürgerrechte in Deutschland allerdings in einem viel größeren Ausmaß rechtlich kodifiziert und werden staatlich sanktioniert.

In Deutschland werden die die industriellen Bürgerrechte etwa durch direkte Mitbestimmungsrechte der Beschäftigten, die Tarifautonomie, die Etablierung der Institution der Betriebsräte sowie die Unternehmensmitbestimmung verkörpert. Die deutsche betriebliche Mitbestimmung geht zurück bis auf die Arbeiterausschüsse im Kaiserreich, fand nach dem Betriebsratsgesetz der Weimarer Republik (1920) erst nach dem 2. Weltkrieg mit dem Betriebsverfassungsgesetz (BetrVG) von 1952 zu der Form, wie wir sie heute kennen (Milert u. Tschirbs 2012; MüllerJentsch 2011). Seit der ersten Fassung des BetrVG von 1952 wurden in seinen Novellierungen (z. B. 1972, 1998, 2001) die Partizipations- und Mitbestimmungsrechte der Arbeitnehmer ausgebaut. Mit der Institutionalisierung der Betriebsräte wurde auch eine Institution als Grundlage für den Kampf um die Anerkennung als Betriebsbürger gelegt: Die Betriebsräte sind ein Bindeglied zu überbetrieblichen demokratischen Institutionen (wie dem Tarifvertragswesen), zielen aber auch in ihrer Praxis stets auf „die Anerkennung des Betriebsrats als autonomes und gleichwertiges Vertretungsorgan“, die „zugleich ein Kampf um die Anerkennung des vollwertigen betrieblichen Bürgerstatus der Belegschaft ist" (Kotthoff 1994, S. 179). Der kontinuierliche Ausbau der industriellen Bürgerrechte in Deutschland ließ auch hier einen evolutionistischen Optimismus für die $\mathrm{Zu}$ kunft industriellen Bürgerrechte aufkommen (Müller-Jentsch 2008).

\subsection{Demokratie und Mitbestimmung}

Als „Komplementärfunktion für die herrschende Wirtschaftsordnung “ (Kißler 2007, S. 30) ist die Mitbestimmung Bestandteil der sozialen Marktwirtschaft und der repräsentativen Demokratie. Auch wenn häufig von einer „demokratischen Betriebsverfassung " gesprochen wird, ist die betriebliche Mitbestimmung nicht gleichzusetzen mit Demokratie im Betrieb. ${ }^{4}$ Die bruchstückhafte demokratische Organisation der Betriebe ist stets von dem Primat der unternehmerischen Herrschaft (Deutschmann 2002, S. 126 ff.) überdeterminiert, die sich letztlich aus den Eigentümerrechten speist. Macht im Betrieb ist strukturell asymmetrisch zuguns-

4 Vgl. hierzu die Kritik von Hennis an der Debatte über die Demokratisierung aller Lebensbereiche (Hennis 1973). 
ten des Managements ausgeprägt. Die betriebliche Mitbestimmung lässt sich daher aufgrund der signifikant verschiedenen Ressourcen- und Rechteausstattung nur eingeschränkt als „gleichberechtigtes Mit-Bestimmen“ (Müller-Jentsch 2003, S. 451) charakterisieren, es handelt sich „um ein Konfliktfeld mit ausgeprägter Machtasymmetrie in den Sozialbeziehungen, durchwirkt von einer hierarchischen Organisation“ (Kißler et al. 2011, S. 30), in der es immer nur um die „halbe Macht“ (Wassermann 2002, S. 213) geht. Dies drückt sich auch darin aus, dass das Management in Unternehmen etwa nicht gewählt wird und die meisten wirtschaftlichen Entscheidungen nicht durch ein demokratisches Mehrheitsprinzip geregelt werden (Baglioni 1999, S. 19-20). Deshalb ist die Mitbestimmung auch weniger Element einer Wirtschaftsdemokratie, sondern Element einer sozialen Demokratie, in deren Subsystem Elemente von Beteiligung einfließen.

Der Betriebsrat repräsentiert als demokratisch legitimierte Institution alle Beschäftigten, er wird von ihnen „gebildet, gewählt und kontrolliert“ (MüllerJentsch 2008, S. 181-182). Durch die Teilnahme an Entscheidungsprozessen in einzelnen Themengebieten, die ohne seine Teilhabe einseitig dominiert würden, schränkt der mitbestimmende Betriebsrat die Macht des Unternehmers ein.

Demokratie bildet damit den Regulationsmodus der Mitbestimmung - auf der Basis der industriellen Bürgerrechte. Das gilt erstens für die Verfahren: Die Gremien werden in quasi-parlamentarischen Verfahren gewählt. Die Repräsentation im Betriebsratsgremium richtet sich nach dem Verhältnis der abgegeben Stimmen. ${ }^{5}$ Aber, zweitens, werden die Ressourcen des Betriebsrates über die Regulation des demos bestimmt. Hierfür ist nicht die Größe der gesamten Belegschaft des Betriebes maßgeblich, da hier auch die Arbeitnehmer hinzu zählen, die in den Betrieb eingegliedert sind, aber in keinem Vertragsverhältnis zum Betriebsinhaber stehen (Klebe et al. 2012, S. 165-166). Nur die Zahl der Arbeitnehmer, die bei Erlass des Wahlausschreibens zur Betriebsratswahl regelmäßig beim Betriebsinhaber beschäftigt sind, bestimmt über die Anzahl der Mandate und der Freistellungen (Klebe et al. 2012, S. 143 ff.). Das führt, wie im nächsten Abschnitt gezeigt wird, durch die Veränderung der Betriebsorganisation und Personalpolitik im Finanzmarktkapitalismus zu einer Erosion des demos bei der Mandatsbemessung.

Die gewandelten Unternehmensstrategien im Finanzmarktkapitalismus stellen die eigentlichen Treiber des Wandels der industriellen Staatsbürgerrechte dar. Denn die Betriebsverfassung hat immer zwei Komponenten: Die rechtliche Normierung und gleichermaßen die von ,inner- und außerbetrieblichen Faktoren beeinflusste Sozialordnung eines Betriebs" (Neuloh 1956, S. 35). Diese inner- und außerbetrieblichen Faktoren - in unserem Fall die Unternehmenssteuerung - sind der „dynamische Faktor“ für die Sozialordnung des Betriebs (Milert u. Tschirbs 2012, S. 34).

$5 \mathrm{Zu}$ den genauen Verfahren und den Besonderheiten vgl. Klebe et al. (2012, S. 136 ff.). 


\section{Wandel der industriellen Beziehungen und industrielle Staatsbürgerrechte}

\subsection{Unternehmenssteuerung im Finanzmarktkapitalismus}

Die Tendenz zur Erosion der Mitbestimmung vollzieht sich vor dem Hintergrund der „Krise des demokratischen Kapitalismus“ (Streeck 2013), in der die Finanzmärkte eine dominante Rolle eingenommen haben. Stückweise hat seit der Krise der 1970er-Jahre dabei die fordistische Konstellation an Prägekraft verloren. Der fordistische Kapitalismus mit seinem dichten Netz von staatlichen Regulierungen, einer gemischten Wirtschaft und langfristigen Unternehmensstrategien machte verstärkt seit den 1990er-Jahren - dem Regime des Finanzmarktkapitalismus Platz (Dörre 2009a; Windolf 2005). Der Etablierung des Finanzmarktkapitalismus, die Shareholder-Value-Steuerung von Großunternehmen bis zum Mittelstand und die daraus erwachsenden arbeitspolitischen Folgen gehören zu den wichtigsten industrie- und wirtschaftssoziologischen Debatten der letzten Jahre (Dörre 2013; Brinkmann 2011; Becker 2009; Streeck 2009a; Windolf 2005; Beyer u. Höpner 2003; Höpner 2003; Lehndorff 2009). Die Dynamik der globalen Finanzmärkte fungiert dabei als treibende Kraft für den Wandel der gesellschaftlichen, insbesondere auch der betrieblichen Steuerung hin zu einer stärkeren marktförmigen Logik: „Indem er die Restrukturierungsbemühungen in spezifische Handlungskorridore lenkt und an marktzentrierten Leitbildern und Produktionsnormen ausrichtet, fungiert der Finanzmarktkapitalismus somit tatsächlich als Triebkraft eines nachfordistischen Produktionsmodells" (Dörre u. Brinkmann 2005, S. 87). Die wichtigsten Instrumente der neuen Unternehmenssteuerung im Finanzmarktkapitalismus sind Ergebnisbewertungen, Kennzifferorientierungen und Gewinnvorgaben, die sich an der Kapitalproduktivität orientieren und von den Konzernspitzen auf Geschäftsfelder, Betriebe, Leistungszentren, Profit- oder Costcenter und selbst auf einzelne Arbeitsgruppen herunter gebrochen werden.

Das fordistische Produktionsmodell hatte sich noch durch die relative Abkopplung betrieblicher Abläufe von Marktschwankungen und -risiken ausgezeichnet (Dörre 2009a, 2009b; Brinkmann 2011). Zudem hatte die vergleichsweise große Homogenität und Mobilisierungsfähigkeit der Stammbelegschaften, vor allem in Großunternehmen, den Betriebsräten und Gewerkschaften, einen bedeutenden Einfluss auch auf die Corporate Governance der Unternehmen gestattet - verbunden mit einer Teilhabe an den Produktivitätsgewinnen der Mehrheit im „Normalarbeitsverhältnis“ (Mückenberger 1985) angestellten Stammbeschäftigten. Prekäre Beschäftigung gab es auch zu Zeiten des fordistischen Produktionsmodells, allerdings erstreckte sie sich im Wesentlichen auf Zonen jenseits der großen tariflich abgesicherten Bereiche. Im Verhältnis zur Verbreitung des Normalarbeitsverhältnisses war es eine „marginale Prekarität“ (Dörre 2009b).

Mit der Krise der fordistischen Formation bekam der Tripartismus Risse und das Tarifvertragssystem wurde zunehmend perforiert und dezentralisiert (Streeck 2009a; Lehndorff 2009; Haipeter 2009). Die Shareholder-Value-Orientierung führt zu einem „innerbetrieblichen Wettkampfsystem“ (Dörre 2013), Abläufe werden stärker an den Markt angekoppelt bzw. die „Marktgrenzen“ in die Unternehmen hineingeschoben (Brinkmann 2011). 
Der Betrieb als räumlich und arbeitspolitisch kohärente Einheit verlor dabei seine Leitbildfunktion. Seine Grenzen sind nicht mehr durch die Fabrikmauern definiert, sondern er wandelt sich zu einer fragmentierten Fabrik mit differenzierten Systemen der Wertschöpfung (Durand 2007; Brinkmann 2011), die aus einer Vielzahl halbautonomer Profit-Center, eigenständiger Betriebsstätten mit unterschiedlichen Tarif- und Mitbestimmungsbedingungen und vor allem heterogener und in zunehmendem Maße prekärer Beschäftigungsverhältnisse besteht. Dies erlaubt es dem Management, eine Vielzahl von Risiken auf Vertragspartner und Beschäftige zu externalisieren und mitbestimmungspolitische Hemmnisse zu umgehen (am Beispiel Leiharbeit vgl. Holst et al. 2009).

Kapitalmarktorientierte Unternehmen folgen zudem mittlerweile einer Personalpolitik der „unteren Linie“ (Haipeter 2012; Holst et al. 2009): Das Stammpersonal wird nicht mehr am zyklischen Durchschnitt (Personalpolitik der „mittleren Linie“) ausgerichtet, sondern orientiert sich am Personalbedarf der Kapazitätsuntergrenze des Unternehmens. Die Stammkräfte besetzten in dieser Personalpolitik alle qualifikatorischen Schlüsselpositionen, während man bereits für den Normalbetrieb Fremdpersonal - vor allem Leiharbeiter - beschäftigen muss. Die Leiharbeit erfährt somit einen Funktionswandel: Sie wird nicht mehr zum kurzfristigen Ausgleich von Personal- und Auftragsschwankungen eingesetzt, sondern wird als permanentes Element der Personalplanung „strategisch eingesetzt“ (Holst et al. 2009; Seifert u. Brehmer 2008).

Möglich wurde diese Entwicklung nicht zuletzt durch die Novellierung des Arbeitnehmerüberlassungsgesetzes (AÜG) 2003, durch die die traditionelle Regulierung der Leiharbeit in Deutschland modifiziert wurde. Damit wurde quantitativ und qualitativ das Statusdifferential der Leiharbeiter sichtbar und zum Objekt gesellschaftlicher Kontroversen. Nachdem es den Gewerkschaften seit 2010 immer stärker gelungen ist, große Bereiche der Leiharbeit durch sogenannte „Besservereinbarungen" 6 sowie durch tarifliche Anstrengungen zu verteuern, rückte jüngst der Werkvertrag immer mehr in den Fokus. Von Experten und Branchenvertretern wird bereits eine Substituierung der Leiharbeit durch Werkverträge forciert (FR 12.02.2012; FAZ 13.03.2013). ${ }^{7}$ Ebenso wie die Leiharbeit ist der Werkvertrag zwar kein neues Element der betrieblichen Steuerung, aber er gewinnt in den letzten Jahren eine zunehmende Bedeutung als Instrument der Flexibilisierung und Externalisierung des Risikos (Klein-Schneider u. Beutler 2013). Hier sind die Auswirkungen für die Mitbestimmung teilweise noch größer als bei der Leiharbeit.

\subsection{Erosion der industriellen Bürgerechte}

Indem sich die Einheit der früheren Belegschaft schrittweise zu einem Flickenteppich unterschiedlicher Beschäftigungsverhältnisse in einer Wertschöpfungskette oder der auf einem Betriebsgelände Erwerbstätigen wandelt, modifiziert sich auch

6 Allein im Organisationsbereich der IG Metall gab es in den letzten Jahren mehr als 1400 solcher Vereinbarungen.

7 Dies wird auch in unseren Experteninterviews immer wieder betont (I1, I2, I3). 
der demos der betrieblichen Mitbestimmung. Während Leiharbeiter, wie später diskutiert wird, noch über gewisse Mitbestimmungs- und Vertretungsrechte verfügen, ist der Werkvertrag ein Kaufvertrag nach dem BGB und unterliegt nicht der Betriebsverfassung (bzw. nur der des Werkvertragsunternehmens). Sozial- und Unfallversicherung, Kündigungs- und Mutterschutz, Lohnfortzahlung im Krankheitsfall und nicht zuletzt die Mitbestimmungsrechte werden durch Werkverträge ganz oder teilweise umgangen (Nienhüser u. Baumhus 2002). ${ }^{8}$

Die Erosion der Mitbestimmung durch den strategischen Einsatz von Leiharbeit kommt gewissermaßen durch die Hintertür der betrieblichen Steuerung, denn der Gesetzgeber hatte bei der Novellierung des BetrVG von 2001 eine Konsolidierung, ja teilweise sogar eine Stärkung der Mitbestimmung vorgesehen. Für Kleinund Mittelbetriebe gab es ein vereinfachtes Wahlverfahren, konzernübergreifende Betriebsratsgründungen wurden erleichtert, auch die Zahl der Mandate und Freistellungen wurde maßvoll erhöht. Zwar wurden die Mitbestimmungsrechte kaum gestärkt, aber die Ressourcen der Betriebsräte wenigstens konsolidiert, mitunter sogar leicht ausgeweitet (Däubler 2001; Brinkmann u. Speidel 2006).

Die industriellen Bürgerrechte erodieren nun im Prozess der Unternehmenssteuerung im Finanzmarktkapitalismus auf zwei Weisen: (1) unmittelbar in der Sozialfigur des Leiharbeiters und (2) in den Mitbestimmungsressourcen über die Regulierung des relevanten demos.

(1) Als Sozialfigur ist der Leiharbeiter gekennzeichnet durch immense Statusunterschiede in Fragen der Entgelte gegenüber den Stammkräften mit einem Normalarbeitsverhältnis. Er ist lediglich ein betriebliches Sachmittel und kann sich aufgrund seiner häufig prekären Lebenslage und seiner relativen Desintegration von der Stammbelegschaft sowie den Mobilitätserfordernissen nur schwieriger kollektiv organisieren. Durch die Aufhebung der Beschränkung der Überlassungsdauer und des Synchronisationsverbots wurde der in der Realität ohnehin schwache Kündigungsschutz für Leiharbeiter weiter geschwächt und der quasi-permanente Einsatz von Leiharbeitern ermöglicht, was in den Folgejahren zu einem starken Anstieg der Leiharbeit führte (vgl. Abbildung 2). Dadurch wurde auch ermöglicht, dass Leiharbeiter häufig nicht mehr nur die Tätigkeiten der Randbelegschaft ausüben, sondern tief integriert in den Produktionsprozess die gleichen Tätigkeiten wie die festangestellten Beschäftigten ausführen.

(2) Der demos war lange Zeit durch die Organisationsmitgliedschaft präzise geregelt (Luhmann 1976). Aber erst durch die Heterogenisierung der Beschäftigungsverhältnisse im Finanzmarkkapitalismus erhält der demos eine neue mitbestimmungspolitische Bedeutung. Relevant ist der dem BetrVG zugrundeliegende Betriebs- und Arbeitnehmerbegriff: Wer ein Angehöriger des Betriebs bzw. wer der für die Bemessung der Ressourcen ausschlaggebende demos ist. In der Novellierung des BetrVG von 2001 wurden die Partizipationsrechte von Nicht-VollzeitBeschäftigen gestärkt, sie wurden den Vollzeitkräften mit kleinen Einschränkun-

8 In einer internen Stichprobe bei Betriebsräten der IG Metall in NRW gaben 52\% der Betriebsräte an, dass der Grund zum Einsatz von Werkverträgen die Umgehung der Mitbestimmung ist. 55\% gaben an, dass Werkverträge in ihrem Unternehmen seit 2008 zugenommen haben (Iwanowski 2013). 
gen gleichgestellt - nach einem halben Jahr Betriebszugehörigkeit erhielten sie auch das passive Wahlrecht. Andere Beschäftige im Betrieb - vor allem Leiharbeiter - bleiben aber nur hybride Betriebsbürger. Denn nur wer ein Beschäftigter des Betriebes ist, ist auch ein mit vollen Rechten und Ressourcen ausgestatteter Betriebsbürger. Leiharbeiter erhielten im novellierten BetrVG zwar das aktive Wahlrecht im Entleihbetrieb (nach drei Monaten Betriebszugehörigkeit), aber nicht das passive. Sie konnten also Repräsentanten wählen, aber selbst keine demokratischen Funktionen ausüben. Und obwohl sie wie die Stammkräfte in den Betriebsablauf eingebunden und weisungsgebunden sind, gehörten sie bis zum Urteil des BAG vom 13.03.2013 nicht zum demos des Betriebes, der zur Bemessung der Mandate und Freistellungen hinzugezogen wird (7 ABR 53/02, DB 2003, 2128), da sie juristisch nicht als Arbeitnehmer des Einsatzbetriebs gezählt wurden. ${ }^{9}$ Damit führt der strategische Einsatz von Leiharbeit in vielen Fällen zu einem Mandatsmanko, d.h. die Ressourcen des Betriebsrates sind proportional geringer, weil der relevante demos kleiner ist als die Gesamtbelegschaft.

Geht man ausschließlich von den formal rechtlichen Normen aus, haben sich die Partizipationsrechte von Leiharbeitern auf den ersten Blick verbessert. Im Verleihbetrieb, in dem sie angestellt sind, verfügen sie über das aktive und passive Wahlrecht, im Entleihbetrieb zusätzlich noch über das (eingeschränkte) aktive. Da aber nur in wenigen Verleihbetrieben überhaupt Betriebsräte existieren und da gleichzeitig die Entleihdauer hoch ist - nach einer Umfrage verweilen 69 Prozent der Leiharbeiter mehr als drei Monate in einem Unternehmen (IW 2011) -, sind die Leiharbeiter vor allem in den Betriebsprozess des Entleihunternehmens integriert. Auf Grund seines prekären Status im Entleihbetrieb verfügt er jedoch über verringerte Ressourcen zur Partizipation. ${ }^{10}$

Mit dem Ansatz von Lockwood zur „staatsbürgerlichen Schichtung“ ([1996] 2000, S. 163 ff.) kann man die eingeschränkte betriebliche Staatsbürgerschaft begrifflich fassen. Lockwood ging in seinem Aufsatz davon aus, dass man in den Industriellen Beziehungen ein „staatsbürgerliches Defizit“ vorfindet, da Arbeitnehmer zwar de jure im Vollbesitz gleicher Bürgerrechte sind, jedoch de facto durch die geringere Ressourcenausstattung ein Machtdefizit gegenüber dem Arbeitgeber habe. Dies könne nur mittels der Ausnutzung industrieller Bürgerrechte ausgeglichen werden. Bei der Sozialfigur des Leiharbeiters ist dieses Defizit jedoch von anderer Qualität. Er verfügt sowohl de facto über eine geringere Ressourcenausstattung gegenüber dem Unternehmer - auch nochmals geringer als die Stammkräfte -, als auch auf hybride Weise de jure. Er kann in seinem Einsatzbetrieb (in dem er oft über lange Zeit arbeitet), d. h. seinem de-facto-Unternehmen, das passive Wahlrecht nicht ausüben. Es handelt sich beim Leiharbeiter also nicht nur um eine „fehlende Übereinstimmung von formaler und substanzieller Staatsbürgerschaft" (Lockwood [1996] 2000, S. 166), sondern ihnen bleiben die „vollen Staatsbürgerrechte“ verwehrt, was nach der Kategorisierung von Lockwood

9 Zur Bedeutung des Urteils vgl. das Fazit dieses Aufsatzes.

10 Dies gilt für die institutionelle-betriebliche Ebene. Auf der Ebene der gewerkschaftlichen Mitgliedschaft und tariflichen Eingliederung konnte etwa die IG Metall in jüngster Zeit durchaus Erfolge in den Teilhabemöglichkeiten von Leiharbeitern schaffen. 
der „staatsbürgerlichen Exklusion“ (Lockwood [1996] 2000, S.164-165) gleichkommt. Mit anderen Worten: Der Leiharbeiter ist ein Betriebsbürger zweiter Klasse. Im nächsten Abschnitt werden dazu einige empirische Befunde präsentiert, die diese Überlegungen untermauern.

\section{Ergebnisse der explorativen Datenanalyse}

\subsection{Ergebnisse aus der WSI-Betriebsrätebefragung}

Der Wandel industrieller Staatsbürgerrechte und die Unternehmenssteuerung im Finanzmarktkapitalismus - das ist im Folgenden die Überlegung - führen zu einer Postdemokratisierung der Mitbestimmung, indem sie die Mitbestimmungsstrukturen erodieren lassen. Einschränkend ist vorwegzuschicken, dass die Datenlage zu demokratischen Verfahren im Betrieb sich als bisher wenig hinreichend herausstellt. Die Schwierigkeiten beginnen bereits mit sehr grundlegenden Defiziten wie etwa dem Fehlen eines vollständigen Registers für Betriebs- und Personalräte in Deutschland. Allerdings lassen sich auf der Basis der WSI-Betriebsrätebefragungen durchaus Auswirkungen der Leiharbeit auf die Mitbestimmungsstrukturen untersuchen. Weil quantitative Datensätze zur betrieblichen Mitbestimmung die Komplexität der mikropolitischen Interaktions- und Machtbeziehungen von Management und Betriebsrat und damit auch deren demokratische Praxis nur unzureichend abbilden können, ergänzen wir im nächsten Abschnitt unsere quantitativen Analysen noch mit einer qualitativen Fallstudie.

Wie oben dargelegt ist der Betriebsrat eine, wenn nicht die zentrale demokratische Institution der Betriebsverfassung. Die Größe des gewählten Gremiums wächst mit der Anzahl der zu vertretenden Beschäftigten, gleiches gilt für die Anzahl der für die Betriebsratsarbeit freigestellten Betriebsräte; in beiden Fällen aber gilt lediglich eine degressive Steigerung, so dass in größeren Betrieben die Zahl der von einem einzelnen (bzw. von einem freigestellten) Betriebsrat zu vertretenden Beschäftigten deutlich höher als in kleineren Betrieben ist.

Wie oben dargelegt, ist in den vergangenen eineinhalb Jahrzehnten eine sinkende Repräsentationsquote durch Betriebsräte festzustellen (vgl. Abbildung 1). Der dynamische Zuwachs atypischer Beschäftigungsverhältnisse (Abbildung 2), insbesondere der Leiharbeit in der Folge der Hartz-Gesetzgebung, wirkt sich nun sowohl auf die formale Ausprägung als auch auf die Aufgaben der gewählten Beschäftigtenvertreter aus. 
Abbildung 2: Entwicklung atypischer Beschäftigung in Deutschland 2006-2011 (in \%)

befristet Beschäftigte —Teilzeitbeschäftigte

- geringfügig Beschäftigte — Leiharbeitskräfte

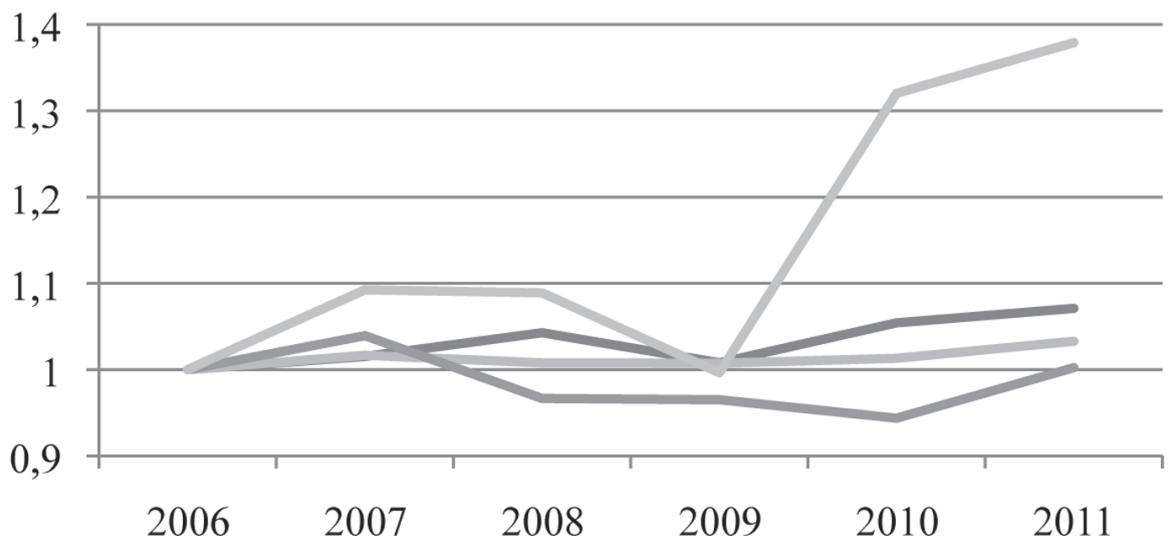

Quelle: Statistisches Bundesamt o. J.; eigene Darstellung.

Blickt man auf das aktive Wahlrecht, so lässt sich anhand der WSI-Betriebsrätebefragung nachzeichnen, dass lediglich 51,2 Prozent der Betriebe, die 2007 LeiharbeitnehmerInnen länger als drei Monate beschäftigten, diese auch in die Wählerverzeichnisse aufgenommen haben. Dies ist ein klares Indiz dafür, dass Leiharbeitskräfte oftmals das aktive Wahlrecht in den Einsatzbetrieben nicht wahrnehmen können, obwohl es ihnen formal zusteht.

Die Zunahme der Leiharbeit bewirkt aber auch ein Abschmelzen der Mitbestimmungsstrukturen an sich, da Leiharbeiter nicht bei der Bemessung der Mandate berücksichtigt werden. ${ }^{11}$ Das daraus entstehende Mandatsmanko entspricht der negativen Differenz zwischen den tatsächlichen Mandatszahlen und der Mandatszahl, die sich ergibt, wenn die überlassenen Beschäftigten zur Bemessung hinzugezählt würden. Hierzu greifen wir auf die Daten der WSI-Betriebsrätebefragung zurück, die allerdings nur für das Jahr 2007 hinreichend vorliegen. Dabei ist auch der Umstand zu beachten, dass zu diesem Zeitpunkt der strategische Einsatz von Leiharbeit noch nicht so fortgeschritten war wie in den späteren Jahren, mit anderen Worten: Die realen Effekte dürften heute über den gemessenen liegen.

11 Der vorliegenden Forschungsarbeit liegen Daten aus der Zeit vor dem BAG Urteil vom 13. März 2013 zugrunde. Erst im Fazit wird auf die veränderte rechtliche Lage eingegangen. 
Tabelle 1: Mandatsmanko

\begin{tabular}{|l|c|}
\hline Mandatsmanko & $\begin{array}{c}2007 \\
\text { relative Häufigkeiten }\end{array}$ \\
\hline Ja & $23,4 \%$ \\
\hline Nein & $76,6 \%$ \\
\hline Gesamt & $100 \%$ \\
\hline
\end{tabular}

Quelle: WSI-Betriebsrätebefragung 2007; eigene Berechnungen (gewichtete Daten); N=805.

Es wird deutlich, dass 23,4 Prozent der im Jahr 2007 befragten Betriebsräte von den Betrieben, die LeiharbeitnehmerInnen einsetzen, mehr Betriebsratsmitglieder hätten, wenn entliehene Arbeitskräfte, die mehr als drei Monate vor Ort beschäftigt waren, zur Bemessung der Betriebsratsgröße hinzugezogen worden wären. Bei den 805 befragten Betriebsräten der Leiharbeitseinsatzbetriebe fehlten auf Grund der Vertretungslücke insgesamt 606 Mandate. Dies hat zur Folge, dass jedes Betriebsratsmitglied quantitativ mehr Beschäftigte betreuen muss.

Eine multiple logistische Regression ${ }^{12}$ fördert zutage, welche Strukturmerkmale den stärksten Einfluss auf die abhängige Variable - das Mandatsmanko - haben. Im Zusammenhang mit dem Mandatsmanko ist davon auszugehen, dass verschiedene Strukturmerkmale einen Einfluss auf sein Auftreten haben. Um dies zu überprüfen, wurden folgende unabhängige Variablen in die Analyse einbezogen:

- Betriebsgründungsjahr (klassiert)

- Betriebsgröße (klassiert $\left.{ }^{13}\right)$

- Wirtschaftsbereich (klassiert)

- Organisationsgrad der Belegschaft (klassiert)

- Inhaberführung

- Anzahl der Ebenen komplexer Gremien.

Anhand der multiplen logistischen Regression wird deutlich, dass die Variablen Betriebsgründungsjahr, Betriebsgröße sowie Inhaberführung einen signifikanten Einfluss auf die Wahrscheinlichkeit des Auftretens eines Mandatsmankos haben (Tabelle 2). Die Wahrscheinlichkeit des Vorkommens eines Mandatsmankos steigt, je jünger das Unternehmen ist, bzw. sinkt mit steigendem Betriebsalter. Ein

12 Sie kommt zur Anwendung, wenn die lineare Regression aufgrund des Skalenniveaus der abhängigen Variablen Mandatsmanko nicht eingesetzt werden kann. Auch auf der unabhängigen Seite bestehen wenige Einschränkungen, die sich, wie bei einer linearen Regressionsanalyse, insbesondere auf ordinale Variablen beziehen, die entweder als kategorial oder als (quasi)metrisch behandelt werden. Zur Interpretation des Einflusses der unabhängigen Variablen auf die abhängige Variable, sind die Signifikanzen, welche die Nullhypothese testen, zu betrachten. Diese Annahme besagt, dass der Einfluss (B) der jeweiligen unabhängigen Variable gleich null ist, diese also keinen Effekt auf die abhängige Variable besitzt (Baltes-Götz 2008). Neben den Signifikanzen ist auch der Effektquotient $\mathrm{e}^{\mathrm{b}}$, auch Odds Ratio genannt, zu prüfen. Dieser gibt den Faktor der Vervielfachung des Wahrscheinlichkeitsverhältnisses (des Odds) an, wenn die Ausprägung der unabhängigen Variablen um eine Einheit erhöht wird (Diaz-Bone u. Künemund 2003). Sowohl die Wirkungsrichtung als auch Wirkungsstärke der unabhängigen Variable wird bei der Betrachtung dieser Kennzahl deutlich (Backhaus et al. 2011).

13 Die Klassierung der Betriebe erfolgte angepasst an die Schwellenwerte bzgl. der Mandatszahlen aus dem BetrVG. 
weiterer Punkt, der die Chance des Auftretens senkt, ist die Inhaberführung der betrachteten Betriebe. Die Wahrscheinlichkeit, dass eine Vertretungslücke durch Nicht-Berücksichtigung von Leiharbeitskräften verursacht wird, steigt zudem mit jeder erreichten höheren Schwelle der Betriebsgröße um das 1,6-fache.

\section{Tabelle 2: Einfluss ausgewählter Strukturmerkmale auf das Mandatsmanko14}

\begin{tabular}{|lcc|c|}
\hline Unabhängige Variablen & $\begin{array}{c}\text { Korrelations- } \\
\text { koeffizient }\end{array}$ & Signifikanzen & $\begin{array}{c}\text { Odds } \\
\text { Ratio }\left(\mathrm{e}^{\mathrm{b}}\right)\end{array}$ \\
\hline Betriebsgründungsjahr &,- 307 &, 001 &, 736 \\
Betriebsgröße &, 422 &, 000 & 1,525 \\
Wirtschaftsbereich &,- 047 &, 198 &, 954 \\
$\begin{array}{l}\text { Organisationsgrad der } \\
\text { Belegschaft }\end{array}$ &,- 099 &, 178 &, 906 \\
$\begin{array}{l}\text { Inhaberführung } \\
\text { Anzahl der Ebenen } \\
\text { komplexer Gremien } \\
\text { Konstante }\end{array}$ &,- 631 &, 002 &, 532 \\
\hline
\end{tabular}

Quelle: WSI-Betriebsrätebefragung 2007; eigene Berechnungen (ungewichtete Daten).

Diese Ergebnisse passen zu den Befunden der Mitbestimmungsforschung. Die Wahrscheinlichkeit einer betrieblichen sowie überbetrieblichen Interessenvertretung ist umso größer, je älter die Firmen sind. Demnach ist davon auszugehen, dass Betriebe mit dieser Eigenschaft in der Mitbestimmung auch stärker aufgestellt sind und somit seltener ein Mandatsmanko vorliegt. Zudem gilt: Gerade in größeren Betrieben werden eher Leiharbeitskräfte beschäftigt, ${ }^{15}$ und diese verweilen auch häufig länger als drei Monate im Einsatzbetrieb. ${ }^{16}$ Genau vor diesem Hintergrund ist zu erwarten, dass ein Mandatsmanko mit geringerer Wahrscheinlichkeit in inhabergeführten Unternehmen vorzufinden ist, da dies häufig kleinere Organisationen sind.

Der Befund, dass eine Vertretungslücke mit steigender Betriebsgröße umso wahrscheinlicher ist, hat jedoch auch einen weitreichenderen qualitativen Effekt. Durch die Grenzziehung im Zusammenhang der Schwellenwerte zur Mandatsbemessung trifft die höhere Wahrscheinlichkeit eines Mandatsmankos jene Betriebsräte, die bereits auf Grund dieser Staffelung ohnehin schon mehr Beschäftigte pro

14 Das Modell erklärt nach Nagelkerkes Pseudo R² 10\% der Gesamtvarianz. Dies spricht für ein annehmbares Modell, wenn man sich daran orientiert, dass Backhaus et al. (2010) ein $\mathrm{R}^{2}$ von 0,5 als sehr gut bewerten. Das kleine $\mathrm{R}^{2}$ kann außerdem darauf zurückgeführt werden, dass versucht wird, zwei eher heterogene Gruppen zu modellieren (Fromm 2012).

15 Zahl der eingesetzten Leiharbeitskräfte nach Betriebsgröße: Gamma=0,673***; Quelle: WSIBetriebsrätebefragung 2007, eigene Berechnung (gewichtete Daten).

16 Zahl der Leiharbeitskräfte, länger als drei Monate in Entleihbetrieb nach Betriebsgröße: Gamma $=0,638^{* * *}$; Quelle: WSI-Betriebsrätebefragung 2007, eigene Berechnung (gewichtete Daten). 
Kopf betreuen. Außerdem führt gerade bei diesen größeren Unternehmen erst eine höhere Zahl an Leiharbeitskräften zum Auftreten einer Vertretungslücke. Somit sind die hier betroffenen Mandatsinhaber quasi einer doppelten Pro-Kopf-Belastung ausgesetzt - eine Art der Aushöhlung demokratischer Partizipationschancen durch Überlastung der Repräsentanten.

Um weitere Regelmäßigkeiten aufzudecken, wurde das Mandatsmanko mit weiteren relevanten Variablen korreliert. Dabei bestätigten diese Korrelationen die bisherigen Befunde (Tabelle 3).

\section{Tabelle 3 Charakterisierung der Betriebe mit einem Mandatsmanko ${ }^{17}$}

\begin{tabular}{|l|c|}
\hline \multicolumn{2}{|c|}{ Mandatsmanko } \\
\hline $\begin{array}{l}\text { Kam es durch den Einsatz von LeiharbeitnehmerInnen } \\
\text { zu Auseinandersetzungen mit der Verleihfirma? }\end{array}$ & $0,104^{* *}$ \\
$\begin{array}{l}\text { Welche Unterstützung leisten die Gewerkschaften und } \\
\text { ihre VertreterInnen dem Betriebsrat? }\end{array}$ & $0,066^{*}$ \\
$\begin{array}{l}\text { Gewerkschaftliche Schulungen/Seminare } \\
\text { Organisationsgrad der Mitarbeiter }\end{array}$ & $0,239^{*}$ \\
$\begin{array}{l}\text { Sind Leiharbeitskräfte bei täglicher oder wöchentlicher } \\
\text { Arbeitszeit schlechter gestellt? }\end{array}$ & $0,099^{*}$ \\
$\begin{array}{l}\text { Arbeitsaufwand des Betriebsrats bei Leiharbeitskräften } \\
\text { gegenüber der Stammbelegschaft. }\end{array}$ & $0,110^{*}$ \\
\hline
\end{tabular}

Quelle: WSI-Betriebsrätebefragung 2007, eigene Berechnungen (gewichtete Daten). Erläuterung:

*:Sig.0,05; ${ }^{* *}$ : Sig.0,01; ${ }^{* * *}$ Sig.0,001.

Sofern überlassene Beschäftigte in einem Unternehmen in Bezug auf die wöchentliche oder tägliche Arbeitszeit schlechter gestellt sind, tritt eher ein Mandatsmanko auf. Betriebsräte, die mit einem Mandatsmanko konfrontiert sind, bewerten den Arbeitsaufwand, den sie für Leiharbeitskräfte im Zuge ihrer Sprechstunden betreiben, als eher größer im Verhältnis zum Arbeitsaufwand, der für regulär Beschäftigte betrieben wird. Außerdem ist eher kein Mandatsmanko vorzufinden, wenn durch den Einsatz von entliehenen Mitarbeitern bereits ein Konflikt mit den Verleihfirmen vorkam. Weitere Faktoren sind das Anbieten von Schulungen und Seminaren für die Betriebsräte durch die Arbeitnehmerverbände oder ein hoher Organisationsgrad der Mitarbeiter. Ist dies der Fall, gibt es seltener ein Mandatsmanko - dies verweist darauf, dass der Verfall der industriellen Staatsbürgerrechte in mikropolitischen Machtauseinandersetzungen zumindest lokal aufgehalten werden kann.

Die Postdemokratisierung der Mitbestimmungsstrukturen drückt sich in gleicher Weise auch im Hinblick auf die Freistellungen und das Freistellungsmanko (Tabelle 4) aus.

17 Es werden nur signifikante Ergebnisse ausgewiesen. 
Tabelle 4: Freistellungsmanko

\begin{tabular}{|l|c|}
\hline Freistellungsmanko & $\begin{array}{c}2007 \\
\text { relative Häufigkeiten }\end{array}$ \\
\hline $\mathrm{Ja}$ & $22,5 \%$ \\
Nein & $77,5 \%$ \\
Gesamt & $100 \%$ \\
\hline
\end{tabular}

Quelle: WSI-Betriebsrätebefragung 2007; eigene Berechnungen (gewichtete Daten); N=663.

Insgesamt hätten 22,5 Prozent der Betriebe einen Anspruch auf mehr Freistellungen, sofern Leiharbeitskräfte, die länger als drei Monate im Entleihunternehmen tätig sind, bei Ermittlung dieser Ressource berücksichtigt würden.

Insgesamt verdeutlicht die Analyse der Freistellungslücke ähnliche Ergebnisse wie die des Mandatsmankos - aus Platzgründen wird hier auf eine Detaildarstellung der Analysen verzichtet. Interessant ist, dass in Betrieben mit aktiven Betriebsräten, d. h. in Betrieben mit vielen Betriebsvereinbarungen und vielen Anrufungen der Einigungsstellen sowie signifikant mehr Kontaktmöglichkeiten zur Belegschaft, das Freistellungsmanko eher seltener zu finden ist. Zudem gilt: Je mehr Unterstützungsangebote es seitens der Gewerkschaft gibt und je stärker die Belegschaft gewerkschaftlich organisiert ist, umso seltener tritt eine Lücke bezüglich der Freistellungen auf. Selbiges gilt für die Zahl an gewerkschaftlichen Schulungen: Je häufiger an Schulungen teilgenommen wurde, umso seltener tritt das Manko auf. Betriebe ohne Freistellungslücke sind eher an einen Branchentarifvertrag und Betriebe mit einer Differenz sind eher nicht an einen Tarif gebunden.

Mit Blick auf die Prekarisierung von Arbeit lassen sich demnach de jure markante Einschränkung betrieblicher Partizipation an demokratischen Verfahren und deutliche Schwächungen der politischen Institutionen feststellen. De facto auch das zeigen die empirischen Analysen z. B. hinsichtlich der Wählerlisten wird dieses Niveau real nochmals unterschritten. Und schließlich findet eine schleichende inhaltliche Überlastung der gewählten Vertretungsgremien statt, denn mit dem Anwachsen entstandardisierter Arbeitsverhältnisse steigen die Anforderungen an die betriebliche Interessenvertretung auch qualitativ hinsichtlich neuer betrieblicher Integrations- und Regulierungsprobleme (z. B. im Arbeits- und Gesundheitsschutz bei Leiharbeitskräften; vgl. Becker et al. 2012) und Unsicherheiten der Interessenvertretungen über die Zuständigkeitsbereiche (Ent- oder Verleiher).

Festzuhalten ist: Für den Betriebsrat des Entleihbetriebs entsteht eine Vielzahl neuartiger und komplexer Handlungsanforderungen, unklarer Zuständigkeiten und eine Heterogenisierung der Belegschaft - bei gleichzeitig tendenziell schrumpfenden Ressourcen. 


\section{Abbildung 3: Arbeitsaufwand des Betriebsrats bei Leiharbeiterlnnen gegenüber der Stammbelegschaft (in \%)}

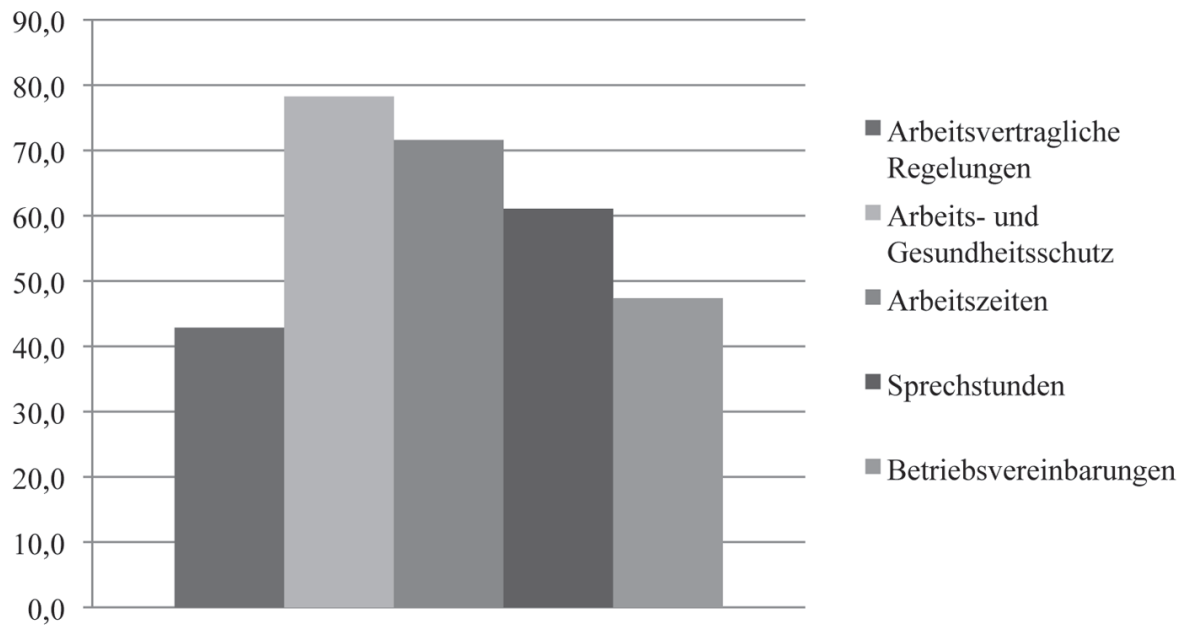

Quelle: WSI-Betriebsrätebefragung 2007; eigene Berechnungen (gewichtete Daten).

Anhand der Daten des WSI-Betriebsrätebefragung wird deutlich, dass je nach Arbeitsbereich zwischen 42,8 Prozent und 78,3 Prozent der Betriebsräte angeben, mindestens den gleich großen oder einen größeren Arbeitsaufwand für die LeiharbeitnehmerInnen im Verhältnis zu den Stammbeschäftigten zu betreiben (Abbildung 3).

\subsection{Eine Fallstudie aus der Automobilindustrie ${ }^{18}$}

Die Automobilindustrie ist in Deutschland die wichtigste exportorientierte Industriebranche und nimmt seit jeher eine Vorreiterfunktion für die Entwicklung von Arbeitsbeziehungen ein (Haipeter 2008). Aber gerade in dieser Branche hat sich durch zahlreiche Modernisierungen des Produktionsprozesses die vertikale Integration der Wertschöpfung stark gewandelt; gleichzeitig wurden die innerbetrieblichen Beschäftigungssysteme über die Integration von Leiharbeit und Werkverträgen stark flexibilisiert (Holst et al. 2009). Die Postdemokratisierung im Unternehmen der Fallstudie zeichnet sich durch die strategische Verknüpfung von atypischer Beschäftigung und Werkverträgen sowie die Fragmentierung der Mitbestimmung aus.

18 Diese Fallstudie beruht auf der wiederholten Untersuchung des Fallbetriebes zwischen 2008-2013. 


\subsubsection{Grenzziehungen in der fragmentierten Fabrik}

Der untersuchte Fallbetrieb stellt das jüngste von mehreren Fertigungswerken eines deutschen Automobilherstellers dar. 2005 in Betrieb genommen wurden Betriebsgelände und Betriebsorganisation bereits im Geiste der modernen Unternehmenssteuerung des Finanzmarktkapitalismus konzipiert. Das Personalkonzept sah von Beginn an eine permanente Leiharbeitsquote von ca. 30 Prozent im Sinne der skizzierten Personalpolitik der unteren Linie vor, das Betriebsgelände bot genug Raum für integrierte Fremdfirmen (Werkverträge), die in den Produktionsprozess eingebunden waren.

„Man hat die Planung so ausgerichtet, dass sie nicht mehr alle Tätigkeiten, die man sonst in den anderen deutschen Werken selbst macht, auch weiterhin eigenbetrieben macht, sondern sofort Tätigkeiten ausgelagert. [...] Die externen Firmen haben die Hallen gebaut gekriegt, haben die Anlagen hineingestellt gekriegt, die haben einen ganz geringen Mietpreis und dürfen da drinnen produzieren“ (I4).

Die Fremdfirmen sind aber nicht nur als Zulieferer auf dem Werksgelände tätig, sondern über Just-in-Sequence-Steuerung unmittelbar in den Produktionsprozess integriert. Einige übernehmen die Montage ganzer Teile (z. B. der Achsen), andere sind für die Logistik in den Werkshallen des fokalen Unternehmens zuständig. Die Logistik ist hierbei integrierter „Teil des Produktionsprozesses“ (I3).

Analytisch lassen sich beim Untersuchungsbetrieb vier Grenzverläufe unterscheiden, die sich mit der Ausnahme von (1) in den Fremdfirmen auf dem Gelände wie ein Fraktal reproduzieren:

(1) Physische Grenze: Die physische Grenze ist das Betriebsgelände, auf dem die Kernproduktion stattfindet. Aber auch außerhalb des Werkes, ein klassisches Greenfield-Werk, befinden sich Zulieferer, die aber nicht unmittelbar in die Betriebsabläufe eingegliedert sind.

(2) Marktgrenze: Im Produktions- und Personalkonzept des Werkes verläuft eine „Marktgrenze“ (Brinkmann 2011), an der sich die klassische Entscheidung „Make or Buy “ vergegenständlicht: Hinter dieser Grenze werden Produktion und Personaleinsatz über externe Marktanbieter reguliert, hier trägt das fokale Unternehmen kein unternehmerisches Risiko. Häufig verläuft die Marktgrenze mitten durch die Produktionsabläufe, sodass Leiharbeiter und Werkvertragsnehmer Seite an Seite mit den Stammbeschäftigten arbeiten. Nur für letztere bedeutet die Marktgrenze die vollständige Inklusion in die arbeitspolitischen und tariflichen Normen des fokalen Unternehmens.

(3) Mitbestimmungsgrenze: Diese Grenzziehungen markiert einerseits den Bereich, in dem der relevante demos konstituiert wird, hier stimmt sie weitgehend mit der Marktgrenze überein (Ausnahme: aktives Wahlrecht der Leiharbeiter). Bei den Mitbestimmungsrechten des Betriebsrates reicht sie jedoch über die Marktgrenze hinaus, da der Betriebsrat des fokalen Unternehmens nach dem BtrVG und den Tarifvereinbarungen etwa auch über Mitbestimmungsrechte beim Einsatz von Leiharbeit verfügt.

(4) Flexibilitätsgrenze: Sie markiert den Bereich, der es dem Betrieb erlaubt, ohne große Friktion bei Marktschwankungen die Produktion zu drosseln und 
Werkverträge und Leiharbeiter kurzfristig auszusteuern, ohne etwa über Sozialpläne oder Abfindungen verhandeln zu müssen. Marktgrenze, Mitbestimmungsgrenze und Flexibilitätsgrenze liegen aber nicht zwangsläufig übereinander. Die Flexibilitätsgrenze wird von der Produktionsorganisation bestimmt. Denn Fremdfirmen und Leiharbeit sind, wie weiter unten ausgeführt wird, so tief in den Produktionsprozess integriert, dass sie nicht immer vollständig abbestellt werden könnten.

\subsubsection{Fragmentierter demos und fragmentierte Mitbestimmung}

In einem idealtypischen Großbetrieb der fordistischen Ära gab es zwar auch Randbelegschaften, aber die gesamte Belegschaft unterlag einem Tarifvertrag und wurde von einem Betriebsratsgremium vertreten, das für die gesamte Belegschaft über Mitbestimmungsrechte verfügte. In unserem Untersuchungsfall handelt es sich zwar de facto um eine Belegschaft, die aber de jure fragmentiert ist. Die Folge ist eine Verringerung der Mitbestimmungsressourcen und der Mitbestimmungsrechte des Betriebsrats des fokalen Unternehmens. Zwar haben mittlerweile viele der größeren Fremdfirmen (für die kleinen gilt dies nicht) eigene Betriebsratsgremien, aber die Interessenvertretung bleibt in und zwischen diesen häufig fragmentiert. Systematische Absprachen zwischen den vereinzelten Gremien finden kaum statt, es gibt keine machtvollen Parallelstrukturen zur Wahrnehmung der industriellen Bürgerrechte gegenüber den (weiterhin) zentralen Entscheidungen des Managements des Fokalunternehmens. Die Strukturen sind in der Regel nur situativ und netzwerkartig.

$\mathrm{Zu}$ (1): Im Jahr 2013 arbeiten für den Fallbetrieb ca. 4300 Personen, von denen 3250 zur Stammbelegschaft gehören. Die restlichen Mitarbeiter sind über direkte Leiharbeitsverhältnisse beim untersuchten Betrieb beschäftigt. Den Anteil der Leiharbeiter hatten Gewerkschaft und Betriebsrat erst kurz vor dem letzten Untersuchungszeitpunkt über eine Betriebsvereinbarung senken können. Neben diesen Beschäftigten sind auf dem Werksgelände zwischen 30 und 40 Fremdunternehmen mit insgesamt ca. 2000 weiteren Mitarbeitern ansässig, die teilweise per Just-in-Sequence-Steuerung unmittelbar in die Ablaufsteuerung des gesamten Werkes integriert sind. Eine genaue Bestandaufnahme von postdemokratischen Defiziten bezüglich der Mandate und Freistellungen innerhalb des gesamten Betriebes (innerhalb der physischen Betriebsgrenze) steht allein schon deshalb vor der Schwierigkeit, dass weder Betriebsrat noch das Management (!) des Fokalunternehmens über genaue Zahlen der Beschäftigten auf dem Werksgelände verfügen. Das liegt vor allem daran, dass Werkverträge und Leiharbeit zumeist nicht über die Personalabteilungen sondern über den Einkauf abgewickelt werden. Hier gibt es eine nicht unerhebliche Dunkelziffer an Fluktuation, da das fokale Unternehmen bei Fremdfirmen in der Regel nicht den Personaleinsatz steuert, sondern nur das Werk abnimmt.

$\mathrm{Zu}$ (2): Vor dem durch die gute Automobilkonjunktur bedingten Kapazitätsaufbau arbeiteten in dem fokalen Unternehmen des Werks über ca. sieben Jahre mehr oder weniger konstant 2700 Stammbeschäftigte innerhalb der Organisationsgrenzen. Die ca. 1000-1200 Leiharbeiter und 2000 Beschäftigte bei Fremdfirmen 
arbeiten am gleichen Ort aber auf unterschiedlichen Seiten der innerbetrieblichen Marktgrenze. Die Leiharbeiter sind durch betriebliche Equal-Pay-Vereinbarungen den Stammbeschäftigten zwar nominal weitgehend gleichgestellt, aber nicht in das Prämiensystem der Beschäftigten des fokalen Unternehmens einbezogen. Für die Werkvertragsarbeitskräfte gelten ebenfalls nicht die gleichen tariflichen und mitbestimmungsrechtlichen Rahmenbedingungen; sie sind im Verhältnis zum Leiharbeiter nicht mal Betriebsbürger zweiter Klasse, sondern doppelt abgewertet als FastNicht-Betriebsbürger. Erwähnenswert ist in diesem Zusammenhang auch, dass die in den Werkvertragsunternehmen eingesetzten Leiharbeitskräfte hinsichtlich ihrer Arbeitsbedingungen und Entlohnung nochmals schlechter gestellt sind als Leiharbeitnehmer, die direkt im Fallbetrieb arbeiten und von Betriebsvereinbarungen zur Besserstellung der Leiharbeitnehmer profitieren.

$\mathrm{Zu}$ (3): Aufgrund der Belegschaftsgröße bei der letzten Betriebsratswahl 2010 besteht das Betriebsratsgremium im fokalen Unternehmen aus 21 Betriebsräten; diese Anzahl der Mandate basiert auf der Bemessung der Stammbeschäftigten dem relevanten demos - diesseits der Marktgrenze. Hätte man die 1000-1200 Leiharbeitnehmer im fokalen Unternehmen als Arbeitnehmer des Betriebs mitgezählt, wären vier Mandate hinzugekommen. Es gab zwar ein Mandats- aber kein Freistellungsmanko, da der Betriebsrat über eine zusätzliche Betriebsvereinbarung eine weitere Freistellung politisch erkämpft hatte. Die Mitbestimmungsrechte des Betriebsrats - etwa im Arbeits- und Gesundheitsschutz - erstrecken sich nach den Regelungen des BetrVG vielfach auch auf die Leiharbeitnehmer. Im Untersuchungsfall hat das fokale Unternehmen seine Arbeits-, Gesundheits- und Sozialbedingungen für die Stammbeschäftigten 1:1 auf die Leiharbeiter übertragen und sie damit weitgehend integriert. Allerdings bedeuten sie für den Betriebsrat auch einen Mehraufwand, „weil es viel mehr Leute sind“ (I4).

Die Werkvertragsunternehmen lassen sich in zwei Gruppen unterteilen. In der ersten Gruppe handelt es sich um zu 100 Prozent in den Produktionsprozess integrierte Dienstleister. Ein Logistikunternehmen übernimmt in der Werkshalle des fokalen Unternehmens die Bereitstellung des Arbeitsmaterials (Schrauben etc.). Die Wege und Räume des Logistikers sind in der Halle durch eine auf den Boden aufgemalte blaue Linie gekennzeichnet, die die Grenze der Unternehmen kennzeichnet. In der Praxis überschreiten die Beschäftigten der beiden Unternehmen aber häufig die blaue Linie und kooperieren unmittelbar miteinander. ${ }^{19}$ Weil hier Stammbeschäftigte, Leiharbeiter und Beschäftigte unmittelbar kooperieren, verfügt der Betriebsrat auch über einen Einfluss auf den Arbeits- und Gesundheitsschutz der Beschäftigten des Dienstleisters. Allerdings arbeiten sie unter völlig unterschiedlichen tariflichen Normen und zu ebenso unterschiedlichen Niveaus industrieller Staatsbürgerrechte.

Anders bei der zweiten Gruppe von Fremdfirmen, die in eigens angemieteten Hallen auf dem Werksgelände arbeiten. Hier verläuft eine klare Mitbestimmungsgrenze zwischen den Hallen: Auf ihre Arbeitsbedingungen hat der Fokal-Betriebsrat daher keinerlei Einfluss. Zwar hat er einen ungefähren Überblick über die

19 Bei einem Werkvertrag sind die Beschäftigten des Werkvertragsnehmers de jure nicht weisungsgebunden gegenüber Beschäftigten des Werkvertragsgebers. 
Anzahl der vom Management abgeschlossenen Werkverträge, aber er hat keine Kenntnis über die genaue Anzahl der eingesetzten Beschäftigten der Werkvertragsnehmer, auch kann er - wie in seinem Unternehmen - die Betriebshallen der Fremdfirmen nicht ohne vorherige Anmeldung betreten und dort ungehindert etwa die Einhaltung des Arbeits- und Gesundheitsschutzes überprüfen.

$\mathrm{Zu}$ (4): Im Zuge der Wirtschaftskrise 2008 wurden fast alle Leiharbeiter im Betrieb ausgesteuert: „Alle abgemeldet [...] und dann auch rausgeschmissen von den [Verleih-]Firmen" (I4). Von den Stammbeschäftigten wurde niemand entlassen. Durch diese Maßnahme konnte die gesamte Stammbelegschaft des Fokalunternehmens in der Krise gesichert werden. Die bestehenden Verträge zu den Werkvertragspartnern wurden fortgesetzt, da sie auch für eine Produktion unter der Normalkapazität funktional notwendig waren. Die Fremdfirmen im Betrieb verfügten allerdings ihrerseits über ähnliche interne Flexibilitätsgrenzen:

„Die haben halt auch ihre Leiharbeiter rausgeworfen, weil die fahren dasselbe Konstrukt, was [das Unternehmen] hier vorgibt. Die haben also auch 30 Prozent Leiharbeitsanteil und die haben stellenweise auch noch Befristungen gehabt" (I4).

\subsubsection{Mitbestimmung und Fremdvergabe: Postdemokratisierungskaskade}

Am Beispiel eines der zentralen Werkvertragsunternehmen kann eine tiefere Verkettung der Postdemokratisierung verdeutlicht werden. Das fokale Unternehmen hat über einen Werkvertrag die Achsenproduktion an den Dienstleister, selbst Tochter eines deutschen Großkonzerns, fremdvergeben. Dieser wiederum wickelt die Produktion der Achsen über Leiharbeitsfirmen ab. Hierbei entstehen mehrere kaskadenförmige Mitbestimmungseinschränkungen, weil auch im Fremdunternehmen die Markt- und Mitbestimmungsgrenzen ihre problematischen Wirkungen entfalten.

Das Dienstleistungsunternehmen produziert die Achsen für zwei fokale Unternehmen. Bei dem einen Unternehmen produziert es außerhalb, beim anderen dem hier untersuchten Fallbetrieb - integriert auf dem Werksgelände. Der Dienstleister selbst hat nur 34 Stammbeschäftigte (und zehn Leiharbeiter aus dem konzerneigenen Verleihbetrieb). Diese Beschäftigten sind vor allem Ingenieure und höhergestellte Angestellte. Die eigentliche Produktion wird wiederum durch ca. 470 Leiharbeiter ausgeführt, von denen 270 für das untersuchte Fokalunternehmen arbeiten. Insgesamt werden die Leiharbeiter von sieben Verleihunternehmen gestellt, wovon allerdings etwa 350, also die große Mehrzahl, von einem Verleihunternehmen gestellt werden, von denen wiederum 210 im Fallbetrieb eingesetzt werden. Dabei ist wichtig zu betonen, dass die große Mehrzahl der Leiharbeiter permanent, also seit 2005, in der Achsenproduktion eingesetzt wird.

Im Jahr 2006 wurde im Dienstleistungsunternehmen ein Betriebsrat gewählt, ein Gremium aus drei Personen, das sich nach der geringen Zahl der Stammbeschäftigten richtete und die Leiharbeiter bei der Bemessung ausschloss. Denn sie durften zwar mitwählen, aber selbst nicht zur Wahl antreten. Die Folge dessen war, dass die Kandidaten für den Betriebsrat sich ausschließlich aus den Ingenieuren und gehobenen Angestellten der Stammkräfte rekrutierten, die aber „mehr von der Arbeitgeberseite“ (I5) stammten. Obwohl sie faktisch, also was die physi- 
sche Anwesenheit innerhalb einer Lokalität angeht, die große Mehrheit im Unternehmen stellten, konnten die Leiharbeiter aufgrund des fehlenden passiven Wahlrechts keine eigenen Interessenvertreter im Werkvertragsunternehmen wählen.

Den Beschäftigten des größten Verleihunternehmens gelang es jedoch, einen eigenen Betriebsrat zu gründen. Sie konnten vor allem bessere Tarifbedingungen an ihrem Standort erreichen. Aber für die schlechteren Arbeitsstandards - „Man strebt immer das unterste Level an [...] Wir arbeiten ein bisschen primitiver" (I5) - im Verhältnis zum fokalen Unternehmen fehlte ihnen die Handhabe. Denn als Betriebsrat des Verleihunternehmens hatten sie durch das BetrVG an ihrem permanenten Arbeitsort im Entleihbetrieb nur eingeschränkte Möglichkeiten:

„[D]as klassische Betriebsverfassungsgesetzt nützt uns im Endeffekt nicht viel. Da stehen zwar viele schöne Sachen drin, aber ich kann sie nicht anwenden, weil das ja nicht in meinem Betrieb ist [...] Ich könnte es bei uns in der Niederlassung anwenden und das war es" (I5).

Die Mitbestimmungsgrenze verläuft also für die Betriebsräte durch ihren eigenen Arbeitsplatz: Sie können nur eingeschränkt ihre Mitbestimmungsrechte dort einsetzen, wo sie de facto tätig sind.

\section{Fazit}

Der vorliegende Beitrag analysiert den Wandel der industriellen Staatsbürgerechte und ihre Auswirkungen auf die betriebliche Mitbestimmung. Der Optimismus, dem man der Entwicklung der Staatsbürgerrechte viele Jahre beimaß, hat sich nicht bestätigt. Im Finanzmarktkapitalismus haben sich die industriellen Staatsbürgerrechte hybridisiert, in der Realität häufig verschlechtert. Dies konnte durch die an T. H. Marshall orientierte, eher formelle Betrachtung der Staatsbürgerrechte gezeigt werden. Die Postdemokratisierung erschließt sich jedoch erst durch eine an Crouch orientierte Analyse informeller Prozesse - neue Unternehmensteuerung, verstärkter Einsatz von prekärer Beschäftigung -, die in der Praxis der Mitbestimmung wirken.

Die formelle Geltung der industriellen Staatsbürgerrechte hat sich durch den strategischen Einsatz von atypischer Beschäftigung de facto in vielen Dimensionen verringert. Die Fragmentierung der Industriellen Beziehungen auf dem Betriebsgelände im Komplex mit dem Wandel der industriellen Bürgerrechte führt auch zu einer Postdemokratisierungskaskade der Fragmentierung und verringerten Reichweite der Mitbestimmung.

Die Gerichte werden deshalb vermehrt zu einer Instanz, die die Postdemokratie in normalen Betriebsablauf - so etwa bei der Stärkung der parlamentarischen Rechte durch das BVG bei den Entscheidungen zum Eurorettungsschirm - bremsen. Zwar vollzog auch das Bundesarbeitsgericht in den letzten Jahren den Liberalisierungsprozess in den Industriellen Beziehungen gebremst nach (Rehder 2011), aber zuletzt traf es eine Reihe von regulierenden Entscheidungen bezüglich der Leiharbeit. Am 13.03.2013 (7 ABR/6911) entschied es, dass Leiharbeiter künftig bei der Bemessung des relevanten demos in Zukunft berücksichtig werden müssen. Damit schränkt das BAG die postdemokratische Tendenz des strategischen Einsatzes von Leiharbeit in vielen Betrieben ein. Allerdings wurde die hyb- 
ride industrielle Bürgerschaft der Leiharbeiter nur zur Hälfte korrigiert. Das passive Wahlrecht erhalten sie auch bei einem permanenten Einsatz im Verleihbetrieb nicht. Auf der anderen Seite wurde gerade der hybride Status des Leiharbeiters fortgeschrieben: Denn Leiharbeiter sind nun ein doppelter relevanter demos: einmal im Ver- und einmal im Entleihbetrieb.

Es ist allerdings zu befürchten, dass der Beschluss des BAG dazu führen wird, dass Leiharbeit in Zukunft noch stärker durch Werkverträge substituiert wird. Dies würde der Fragmentierung der betrieblichen Organisation und damit der Mitbestimmung sogar noch Vorschub geleistet.

\section{Literatur:}

Artus, Ingrid. 2008. Interessenhandeln jenseits der Norm. Mittelständische Betriebe und prekäre Dienstleistungsarbeit in Deutschland und Frankreich. Frankfurt/New York: Campus Verlag.

Backhaus, Klaus, Bernd Erichson, Wulff Plinke und Rolf Weiber. 2011. Multivariate Analysemethoden. Berlin und Heidelberg: Springer.

Baglioni, Guido. 1999. Ist Demokratie möglich? Arbeitnehmerbeteiligung im Unternehmen: Der schwierige Weg zwischen Demokratie und Effizienz. Baden-Baden: Nomos.

Balte-Götz, Bernhard. 2008. Logistische Regressionsanalyse mit SPSS. http://www.uni-trier. de/fileadmin/urt/doku/logist/logist.pdf. Zugegriffen: 21.05.2012.

Barbalet, Jack. 1998. Citizenship. Rights, Struggle and Class Inequality. Milton Keynes: Open University.

Becker, Karina, Ulrich Brinkmann und Thomas Engel. 2008. „Hybride Beteiligung“ im Betrieb? Sachkundige Beschäftigte in der Betriebsratsarbeit. WSI-Mitteilungen 61: 305311.

Becker, Karina. 2009. Die Bühne der Bonität. Wie mittelständische Unternehmen auf die neuen Anforderungen des Finanzmarkts reagieren. Berlin: Edition Sigma.

Benthin, Rainer, und Ulrich Brinkmann (Hrsg.). 2008. Unternehmenskultur und Mitbestimmung. Betriebliche Integration zwischen Konsens und Konflikt. Frankfurt/New York: Campus Verlag.

Beyer, Jürgen, und Martin Höpner. 2003. The Disintegration of Organized Capitalism: German Corporate Governance in the 1990s. West European Politics 26: 179-198.

Brinkmann, Ulrich. 2011. Die unsichtbare Faust des Marktes. Betriebliche Kontrolle und Koordination im Finanzmarktkapitalismus. Berlin: Edition Sigma.

Brinkmann, Ulrich, und Frederic Speidel. 2006. Hybride Beteiligungsformen am Beispiel „sachkundiger Arbeitnehmer“. WSI-Mitteilungen 59: 86-91.

Castel, Robert. 2011. Die Krise der Arbeit. Neue Unsicherheiten und die Zukunft des Individuums. Hamburg: Hamburger edition.

Castel, Robert, und Klaus Dörre (Hrsg.). 2009. Prekarität, Abstieg, Ausgrenzung. Die soziale Frage am Beginn des 21. Jahrhunderts. Frankfurt/New York: Campus Verlag.

Crouch, Colin. 1998a. Staatsbürgerschaft und Markt. Das Beispiel der neueren britischen Bildungspolitik. Berliner Journal für Soziologie 8: 453-472

Crouch, Colin. 1998b. The Globalized Economy: End to the Age of Industrial Citizenship? In Advanced Theory of Labour Law and Industrial Relations in a Global Context, Hrsg. Tom Wilthagen, 151-164. Amsterdam: North-Holand. 
Crouch, Colin. 2008. Postdemokratie. Frankfurt a. M.: Suhrkamp.

Coutu, Michel. 2004. Industrial Citizenship, Human Rights and the Transformation of Labour Law: A Critical Assessment of Harry Arthurs' Legalization Thesis. Canadian Journal of Law and Society/Revue Canadienne Droit et Société 19: 73-92.

Dahrendorf, Ralf. 1992. Der moderne soziale Konflikt. Essay zur Politik der Freiheit. München: DTV.

Dahrendorf, Ralf. 1957. Soziale Klassen und Klassenkonflikt in der industriellen Gesellschaft. Stuttgart: Enke.

Däubler, Wolfgang. 2001. Die novellierte Betriebsverfassung. Industrielle Beziehungen 8: 364-378.

Deutschmann, Christoph. 2002. Postindustrielle Industriesoziologie. Theoretische Grundlagen, Arbeitsverhältnisse und soziale Identitäten. Weinheim/München: Juventa.

Diaz-Bone, Rainer, und Harald Künemund. 2003. Einführung in die binäre logistische Regression. Mitteilungen aus dem Schwerpunktbereich Methodenlehre 56. Berlin: Institut für Soziologie, Freie Universität Berlin.

Dörre, Klaus. 2013. Krise des Shareholder Value? Kapitalmarktorientierte Steuerung als Wettkampfsystem. In Entfesselte Finanzmärkte. Soziologische Analysen des modernen Kapitalismus, Hrsg. Klaus Kraemer und Sebastian Nessel, 121-143. Frankfurt/New York: Campus Verlag.

Dörre, Klaus. 2009a. Die neue Landnahme. Dynamiken und Grenzen des Finanzmarktkapitalismus. In Soziologie, Kapitalismus, Kritik. Eine Debatte, Hrsg. Klaus Dörre, Stephan Lessenich und Hartmut Rosa, 21-86. Frankfurt a. M.: Suhrkamp.

Dörre, Klaus. 2009b. Prekarität im Finanzmarkt-Kapitalismus. In Prekarität, Abstieg, Ausgrenzung. Die soziale Frage am Beginn des 21. Jahrhunderts, Hrsg. Robert Castel und Klaus Dörre, 35-64. Frankfurt/New York: Campus Verlag.

Dörre, Klaus, und Ulrich Brinkmann. 2005. Finanzmarktkapitalismus. Triebkraft eines flexiblen Produktionsmodells? Kölner Zeitschrift für Soziologie und Sozialpsychologie. Sonderheft 45: 85-116.

Durand, Jean-Pierre. 2007. Invisible Chain. Constraints and Opportunities in the New World of Employment. Basingstoke: MacMillan.

Frankfurter Rundschau 12. Februar 2012, S. 2.

Frankfurter Allgemeine Zeitung. 13. März 2013, S. 10.

Fudge, Judy. 2005. After Industrial Citizenship. Market Citizenship or Citizenship at Work? Relations Industrielles/Industrial Relations 60: 631-656.

Ganßmann, Heiner. 1993. Sind soziale Rechte universalisierbar? Zeitschrift für Soziologie 22: 385-394.

Giddens, Anthony. [1983] 2000. Klassenspaltung, Klassenkonflikt und Bürgerrechte. In Citizenship - Soziologie der Staatsbürgerschaft, Hrsg. Jürgen Mackert und Hans-Peter Müller, 183-205. Opladen: Westdeutscher Verlag

Gersuny, Carl. 1994. Industrial Rights: A Neglected Facet of Citizenship Theory. Economic and Industrial Democracy 15: 211-226.

Haipeter, Thomas. 2012. Sozialpartnerschaft in und nach der Krise: Entwicklungen und Perspektiven. Industrielle Beziehungen 19: 387-411.

Haipeter, Thomas. 2009. Kontrollierte Dezentralisierung? Abweichende Tarifvereinbarungen in der Metall und Elektroindustrie. Industrielle Beziehungen 16: 232-253. 
Haipeter, Thomas. 2008. The automotive industry in Germany. In The labour impact of globalization in the automotive industry. A comparison of the Italian, German, Spanish and Hungarian motor industries, Hrsg. Paolo Caputo und Elisabetta Della Corte, 61102. Rom: Fondazione G. Brodolini.

Hauser-Ditz, Axel, Markus Hertwig und Ludger Pries. 2008. Betriebliche Interessenregulierung in Deutschland. Arbeitnehmervertretung zwischen demokratischer Teilhabe und ökonomischer Effizienz. Frankfurt/New York: Campus Verlag.

Hennis, Wilhelm. 1973. Demokratisierung. - Zur Problematik eines Begriffs. In ders.: Die mißverstandene Demokratie, 26-51. Freiburg: Herder.

Holst, Hajo, Oliver Nachtwey und Klaus Dörre. 2009. Funktionswandel und Leiharbeit: Neue Nutzungsstrategien und ibre arbeits- und mitbestimmungspolitischen Folgen. Arbeitsheft der Otto-Brenner-Stiftung 61, Fankfurt a. M..

Höpner, Martin. 2003. Wer beherrscht die Unternehmen? Shareholder Value, Managerherrschaft und Mitbestimmung in Deutschland. Frankfurt/New York: Campus Verlag.

Iwanowski, Christian. 2013. Projekt Industrielle Dienstleistungen/Leiharbeit. Beitrag präsentiert beim 9. Hans-Böckler-Forum zum Arbeits- und Sozialrecht, 21. März 2013, Berlin.

IW Consult. 2011. Zeitarbeit in Deutschland. Institut der deutschen Wirtschaft Köln Consult GmbH. Köln: Institut der deutschen Wirtschaft.

Joppke, Christian. 2010. The Inevitable Lightning of Citizenship. European Journal of Sociology 51: 9-32

Joppke, Christian. 2007. Transformation of Citizenship: Status, Rights, Identity. Citizenship Studies 11: 37-48.

Kißler, Leo. 2007. Politische Soziologie. Grundlagen einer Demokratiewissenschaft. Stuttgart: UVK/UTB.

Kißler, Leo, Ralph Greifenstein und Karsten Schneider. 2011. Die Mitbestimmung in der Bundesrepublik Deutschland. Wiesbaden: VS Verlag für Sozialwissenschaften.

Klebe, Thomas, Jürgen Ratayczak, Michael Heilmann und Sibylle Spoo. 2012. Betriebsverfassungsgesetz. Basiskommentar mit Wablordnung. Frankfurt a. M.: Bund.

Klein-Schneider, Hartmut, und Kai Beutler. 2013. Werkvertragsunternehmen. Outsourcing auf dem Betriebsgelände. WSI-Mitteilungen 66: 149-159.

Kotthoff, Hermann. 1994. Betriebsräte und Bürgerstatus. Wandel und Kontinuität betrieblicher Mitbestimmung. München/Mehring: Rainer Hampp.

Lehndorff, Steffen (Hrsg.). 2009. Abriss, Umbau, Renovierung? Studien zum Wandel des deutschen Kapitalismusmodells. Hamburg: VSA.

Lessenich, Stephan. 2008. Die Neuerfindung des Sozialen. Der Sozialstaat im flexiblen Kapitalismus. Bielefeld: transcript.

Lockwood, David. [1996] 2000. Staatsbürgerliche Integration und Klassenbildung. In Citizenship - Soziologie der Staatsbürgerschaft, Hrsg. Jürgen Mackert und Hans-Peter Müller, 157-180. Opladen: Westdeutscher Verlag.

Luhmann, Niklas. 1976. Funktionen und Folgen formaler Organisation. Berlin: Duncker \& Humblot.

Mackert, Jürgen. 2006. Staatsbürgerschaft. Eine Einführung. Wiesbaden: VS Verlag für Sozialwissenschaften. 
Mann, Michael. [1987] 2000. Strategien der herrschenden Klasse und Citizenship. In Citizenship - Soziologie der Staatsbürgerschaft, Hrsg. Jürgen Mackert und Hans-Peter Müller, 207-228. Opladen: Westdeutscher Verlag.

Marshall, Thomas. [1950] 1992. Staatsbürgerrechte und soziale Klassen. In Bürgerrechte und soziale Klassen, Hrsg. Elmar Rieger, 33-93. Frankfurt/New York: Campus Verlag.

Milert, Werner, und Rudolf Tschirbs. 2012. Die andere Demokratie. Betriebliche Interessenvertretung in Deutschland. 1848 bis 2008. Essen: Klartext.

Mückenberger, Ulrich. 1985. Die Krise des Normalarbeitsverhältnisses. Hat das Arbeitsrecht noch Zukunft? Zeitschrift für Sozialreform 7, 415-434 u. 457-474.

Müller-Jentsch, Walther. 2011. Gewerkschaften und Soziale Marktwirtschaft seit 1945. Stuttgart: Reclam.

Müller-Jentsch, Walter. 2008. Arbeit und Bürgerstatus. Studien zur sozialen und industriellen Demokratie. Wiesbaden: VS Verlag für Sozialwissenschaften.

Müller-Jentsch, Walther. 2007. Strukturwandel der Industriellen Beziehungen. ,Industrial Citizenship'zwischen Markt und Regulierung. Wiesbaden: VS Verlag für Sozialwissenschaften.

Müller-Jentsch, Walther. 2003. Mitbestimmungspolitik. In Die Gewerkschaften in Politik und Gesellschaft der Bundesrepublik Deutschland. Ein Handbuch, Hrsg. Wolfgang Schroeder und Bernhard Weßels, 451-477. Opladen: Westdeutscher Verlag.

Neuloh, Otto. 1956. Die deutsche Betriebsverfassung und ihre Sozialformen bis zur Mitbestimmung. Tübingen: J. C. B. Mohr.

Nienhüser, Werner, und Walter Baumhus. 2002. ,Fremd im Betrieb‘. Der Einsatz von Fremdpersonal als Arbeitskräftestrategie. In Neue Formen der Beschäftigung - neue Personalpolitik?, Hrsg. Albert Martin und Werner Nienhüser, 61-120. München/ Mehring: Rainer Hampp.

Parsons, Talcott. [1977] 2000. Gleichheit und Ungleichheit in modernen Gesellschaften. Zur Bedeutung sozialer Schichtung. In Citizenship - Soziologie der Staatsbürgerschaft, Hrsg. Jürgen Mackert und Hans-Peter Müller, 103-129. Opladen: Westdeutscher Verlag.

Parsons, Talcott. 1985. Das System moderner Gesellschaften. Weinheim/München: Juventa Rees, Anthony. 1996. T. H. Marshall and the Progress of Citizenship. In Citizenship today, Hrsg. Martin Bulmer und Anthony Rees, 1-22. London: UCL.

Rehder, Britta. 2011. Rechtsprechung als Politik. Der Beitrag des Bundesarbeitsgerichts zur Entwicklung der Arbeitsbeziehungen in Deutschland. Frankfurt/New York: Campus Verlag.

Roche, Maurice. 1987. Citizenship, Social Theory, and Social Change. Theory and Society 16: 363-399.

Seifert, Hartmut, und Wolfram Brehmer. 2008. Leiharbeit. Funktionswandel einer flexiblen Beschäftigungsform. WSI-Mitteilungen 61: 335-341.

Schroeder, Wolfgang, und Rainer Weinert. 1999. Anmerkungen zum Wandel industrieller Beziehungen in Deutschland. Kontrollierte oder unkontrollierte Dezentralisierung. Zeitschrift für Politikwissenschaft 9: 1295-1317.

Streeck, Wolfgang. 1997. Industrial Citizenship under Regime Competition: the Case of the European Works Councils. Journal of European Public Policy 4: 643-64.

Streeck, Wolfgang. 2009a. Re-Forming Capitalism. Institutional Change in the German Political Economy. Oxford: Oxford University. 
Streeck, Wolfgang. 2009b. Industrial Relations Today. Reining in Flexibility. MPIfG Working Paper 08/3. Köln: Max-Planck-Institut für Gesellschaftsforschung.

Streeck, Wolfgang. 2013. Gekaufte Zeit. Die vertagte Krise des demokratischen Kapitalismus. Berlin: Suhrkamp.

Streeck, Wolfgang, und Anke Hassel. 2003. The Crumbling Pillars of Social Partnership. In Germany. Beyond the Stable State, Hrsg. Herbert Kitschelt und Wolfgang Streeck, 101124. London: Frank Cass.

Streeck, Wolfgang, und Norbert Kluge (Hrsg.). 1999. Mitbestimmung in Deutschland. Tradition und Effizienz. Expertenberichte für die Kommission Mitbestimmung Bertelsmann Stiftung/Hans-Böckler-Stiftung. Frankfurt/New York: Campus Verlag.

Tilly, Charles. 1997. A Primer on Citizenship. Theory and Society 26: 599-602.

Turner, Bryan. 2001. The Erosion of Citizenship. British Journal of Sociology 52: 189-209.

Turner, Bryan. [1990] 2000. Grundzüge einer Theorie der Staatsbürgerschaft. In Citizenship - Soziologie der Staatsbürgerschaft, Hrsg. Jürgen Mackert und Hans-Peter Müller, 230-261. Opladen: Westdeutscher Verlag.

Turner, Bryan. 1986. Citizenship and Capitalism. London: Allen and Unwin.

Wassermann, Wolfram. 2002. Betriebsräte. Akteure für Demokratie in der Arbeitswelt. Münster: Westfälisches Dampfboot.

Windolf, Paul (Hrsg). 2005. Finanzmarktkapitalismus. Analysen zum Wandel von Produktionsregimen. Sonderheft der Kölner Zeitschrift für Soziologie und Sozialpsychologie. Sonderheft 45. Wiesbaden: VS Verlag für Sozialwissenschaften.

\section{Autorenangaben:}

Prof. Dr. Ulrich Brinkmann,

Universität Trier, Lehrstuhl Wirtschaftssoziologie, Universitätsring 15, 54296 Trier, brinkman@uni-trier.de

Dr. Oliver Nachtwey, Universität Trier, Lehrstuhl Wirtschaftssoziologie, Universitätsring 15, 54296 Trier, nachtwey@uni-trier.de [Korrespondenzautor; derzeit Gastwissenschaftler am Institut für Sozialforschung, Frankfurt] 